\title{
On third homologies of groups and of quandles via the Dijkgraaf-Witten invariant and Inoue-Kabaya map
}

\author{
TAKEFUMI NOSAKA
}

\begin{abstract}
We propose a simple method for producing quandle cocycles from group cocycles by a modification of the Inoue-Kabaya chain map. Further, we show that, with respect to "universal extension of quandles", the chain map induces an isomorphism between third homologies (modulo some torsion). For example, all Mochizuki's quandle 3 -cocycles are shown to be derived from group cocycles. As an application, we calculate some $\mathbb{Z}$-equivariant parts of the Dijkgraaf-Witten invariants of some cyclic branched covering spaces, via some cocycle invariant of links.
\end{abstract}

20J06, 57M12; 57M27, 57N65

\section{Introduction}

A quandle is a set $X$ with a binary operation whose definition was partially motivated by knot theory. Fenn, Rourke and Sanderson [8;9] defined a space $B X$, called the rack space in analogy to the classifying spaces of groups. Carter et al $[3 ; 4]$ introduced quandle cohomologies $H_{Q}^{*}(X ; A)$ with local coefficients by slightly modifying the cohomology of $B X$; in addition they defined combinatorially a state-sum invariant $I_{\psi}(L)$ of links $L$ constructed from a cocycle $\psi \in H_{Q}^{*}(X ; A)$. The construction can be considered as an analogue of the Dijkgraaf-Witten invariant [6] of closed oriented 3-manifolds $M$ constructed from a finite group $G$ and a 3 -cocycle $\kappa \in H_{\mathrm{gr}}^{3}(G ; A)$. To be specific, the invariant is defined as the formal sum of pairings expressed by

$$
\mathrm{DW}_{\kappa}(M):=\sum_{f \in \operatorname{Hom}_{\mathrm{gr}}\left(\pi_{1}(M), G\right)}\left\langle f^{*}(\kappa),[M]\right\rangle \in \mathbb{Z}[A],
$$

where $[M]$ is the fundamental class in $H_{3}(M ; \mathbb{Z})$. Inspired by this analogue, for many quandles $X$, Nosaka [22] gave essentially topological meanings of the cocycle invariants, using the Dijkgraaf-Witten invariant and the homotopy group $\pi_{2}(B X)$.

We mainly focus on a relation between quandle and group homologies, which have been investigated in several studies. For example, the second quandle homology has been extensively studied by Eisermann [7] on the basis of the first group homologies. In addition, Nosaka [22] roughly computed some third quandle homologies from the 
group homologies of $\pi_{1}(B X)$ with some ambiguity. Furthermore, for any quandle $X$, Inoue and Kabaya [11] constructed a chain map $\varphi_{\mathrm{IK}}$ from the quandle complex to a certain complex. Although the latter complex seems far from something familiar, Kabaya [13] modified the $\varphi_{\mathrm{IK}}$ mapping to a group homology under a certain strong condition of $X$. Furthermore, for certain special quandles, Nosaka [21] proposed a method for constructing quandle cocycles from invariant theory via their chain map.

This paper demonstrates a relation between third homologies of groups and those of quandles via the Inoue-Kabaya map, with respect to a broad subclass of quandles. Here, a quandle in the subclass is defined as a group $G$ with the operation $g \triangleleft h:=\rho\left(g h^{-1}\right) h$ for $g, h \in G$, where $\rho: G \rightarrow G$ is a fixed group isomorphism (Definition 2.1). Let such a quandle be denoted by $X=(G, \rho)$. Let also $H_{n}^{\mathrm{gr}}(G ; \mathbb{Z})_{\mathbb{Z}}$ be a quotient of the group homology of $G$ subject to the action of $\rho$, called the $\mathbb{Z}$-coinvariant. In Section 2.2, we reformulate the Inoue-Kabaya map, $\Phi_{n}$, which induces a homomorphism

$$
\left(\Phi_{n}\right)_{*}: H_{n}^{Q}(X ; \mathbb{Z}) \longrightarrow H_{n}^{\mathrm{gr}}(G ; \mathbb{Z})_{\mathbb{Z}}
$$

We then lift this map $\Phi_{n}$ to a chain map $\varphi_{n}$ from $C_{n}^{Q}(X ; \mathbb{Z})$ to the usual group homology $H_{n}^{\mathrm{gr}}(G ; \mathbb{Z})$; see Proposition 2.6. As a corollary, if a presentation of a group $n$-cocycle $\kappa$ of $G$ is given, we can easily obtain the presentation of the induced quandle $n$-cocycle $\varphi_{n}^{*}(\kappa)$.

This paper also investigates properties of the chain map $\Phi_{n}$ above. First, we focus on a class of universal quandle coverings $\tilde{Y}$ that are constructed from "connected" quandles $Y$ of finite order. Here $\tilde{Y}$ is a quandle of the form $\left(\operatorname{Ker}\left(\varepsilon_{Y}\right), \rho\right)$ for some group $\operatorname{Ker}\left(\varepsilon_{Y}\right)$, and it possesses an epimorphism $p_{Y}: \tilde{Y} \rightarrow Y$ (as a quandle covering); see Example 2.3 for details. Then we show that the associated chain map $\widetilde{\Phi}_{3}$ induces an isomorphism

$$
\left(\widetilde{\Phi}_{3}\right)_{*}: H_{3}^{Q}(\tilde{Y}) \cong H_{3}^{\text {gr }}\left(\operatorname{Ker}\left(\varepsilon_{Y}\right)\right)_{\mathbb{Z}} \text { up to } t_{Y} \text {-torsion, }
$$

where $t_{Y} \in \mathbb{N}$ is the minimal number satisfying $\rho^{t_{Y}}=\mathrm{id}$ (Theorem 2.10).

Needless to say, $\Phi_{3}$ is not always an isomorphism for such quandles $(G, \rho)$; however, from the universality of coverings, in some cases we can analyze the map $\Phi_{3}$ as follows.

We consider quandles $Y$ of the form $\left(\mathbb{F}_{q}, \times \omega\right)$ with $\omega \in \mathbb{F}_{q}$, referred to commonly as Alexander quandles. Here we regard the finite field $\mathbb{F}_{q}$ as an additive group and the symbol " $\times \omega$ " denotes the $\omega$-multiplication of $\mathbb{F}_{q}$. Fortunately, Mochizuki [19] determined all the quandle 3-cocycles of $Y$, which are the most well-known quandle cocycles so far. However, he found the 3-cocycles of $Y$ by solving certain differential equations over $\mathbb{F}_{q}$, and his statement was not so simple (see Section 4.1). In this paper, 
we easily obtain and explain all his 3-cocycles on the basis of some group 3-cocycles via the map $\Phi_{n}^{*}$ (see (25) and Lemma 4.6). Moreover, we show that the third quandle cohomology $H_{Q}^{3}\left(Y ; \mathbb{F}_{q}\right)$ is isomorphic to a sum of some group homologies via the maps $\Phi_{2}, \Phi_{3}$ and $\widetilde{\Phi}_{3}$ (see Theorem 2.13 in detail). In conclusion, all the Mochizuki 3-cocycles stem from some group 3-cocycles via the three maps. Therefore, such an approach using $\Phi_{*}$ is generally expected to be useful for finding valuable quandle cocycles of the quandles $(G, \rho)$.

As an application of the isomorphism (2), we propose a relation to a partial sum of some Dijkgraaf-Witten invariants of $\widehat{C}_{L}^{t}$, where $\widehat{C}_{L}^{t}$ denotes the $t$-fold cyclic covering space of $S^{3}$ branched over a link $L$ (see (10) for the detailed definition of the partial sum, and denote it by $\left.\mathrm{DW}_{\kappa}^{\mathbb{Z}}\left(\widehat{C}_{L}^{t}\right) \in \mathbb{Z}[A]\right)$. To be specific, we show (Theorem 2.15) that if the induced map $p_{Y}^{*}: H_{Q}^{3}(Y ; A) \rightarrow H_{Q}^{3}(\tilde{Y} ; A)$ is surjective, and if $Y$ is connected and of finite order, then any group 3-cocycle $\kappa$ of the above group $\operatorname{Ker}\left(\varepsilon_{Y}\right)$ admits some quandle 3-cocycle $\psi$ of $Y$ for which the equality

$$
\operatorname{DW}_{\kappa}^{\mathbb{Z}}\left(\hat{C}_{L}^{t}\right)=I_{\psi}(L) \in \mathbb{Z}[A]
$$

holds. Here $I_{\psi}(L)$ is the quandle cocycle invariant of links $L$ [4] (see Remark 2.16 for some quandles satisfying the assumption on $p_{Y}^{*}$ ). While the equivalence of the two invariants was implied in the previous paper [22] by abstract nonsense and the proofs of (2) and (3) are based on some results in [22], the point of our results is that the cocycle $\psi$ is directly obtained from the chain map $\widetilde{\Phi}_{3}$.

Compared with [22], we emphasize that our theorem serves to compute some parts of the Dijkgraaf-Witten invariants $\mathrm{DW}_{\kappa}^{\mathbb{Z}}\left(\widehat{C}_{L}^{t}\right)$ via the right invariant $I_{\psi}(L)$. A standard way to compute the invariant is to find a fundamental class from a triangulation of $M$ (see Dijkgraaf and Witten [6] and Wakui [23]). However, presentations of group 3 -cocycles are intricate in general. Hence most known computations of the DijkgraafWitten invariants are those with respect to abelian groups. However, in computing them via the right invariant $I_{\psi}(L)$, we use no triangulation of $M$ and many quandle 3 -cocycles are simpler than group ones (in our experience).

In fact, in Section 5, we succeed in computing some of the formal sums $\operatorname{DW}_{\kappa}^{\mathbb{Z}}\left(\hat{C}_{L}^{t}\right)$ by using the Mochizuki 3-cocycles, which are derived from triple Massey products of a meta-abelian group $G_{X}$ (see Proposition 4.7). For example, we will calculate the cocycle invariants of the torus knots $T(m, n)$ (see Theorem 5.1); hence, we obtain the partial sum $\mathrm{DW}_{\kappa}^{\mathbb{Z}}$ of the Brieskorn manifold $\Sigma(m, n, t)$, which is the covering space branched over the knot $T(m, n)$. As the special case $\omega=-1$, we compute the cocycle invariant of some knots $K$ and thus obtain some values $\mathrm{DW}_{\kappa}^{\mathbb{Z}}\left(\widehat{C}_{K}^{t}\right)$ for the double covering spaces branched along $K$ (see Table 1 in Section 5.1). 
This paper is organized as follows. In Section 2, we introduce a lift of the InoueKabaya chain map and state our theorems. In Section 3, we prove Theorems 2.10 and 2.15. In Section 4, we show that Mochizuki 3-cocycles are derived from some group 3-cocycles. In Section 5, we calculate some partial sums of the Dijkgraaf-Witten invariants.

Notation and convention $\mathbb{F}_{q}$ is a finite field of characteristic $p>0 . H_{n}^{\mathrm{gr}}(G)$ denotes the group homology of a group $G$ with trivial integral coefficients. We assume that manifolds are smooth, connected and oriented.

\section{Results}

In Section 2.3 and 2.4, we state our theorems. For this purpose, we briefly review quandle homologies and their properties in Section 2.1, and we modify the InoueKabaya map in Section 2.2.

\subsection{Review of quandles and quandle cohomologies}

We start by recalling basic concepts about quandles. A quandle is a set $X$ with a binary operation $(x, y) \rightarrow x \triangleleft y$ such that, for any $x, y, z \in X, x \triangleleft x=x$, $(x \triangleleft y) \triangleleft z=(x \triangleleft z) \triangleleft(y \triangleleft z)$ and there exists a unique $w \in X$ such that $w \triangleleft y=x$. A quandle $X$ is said to be of type $t_{X}$ if $t_{X}>0$ is the minimal number $N$ satisfying $a=(\cdots(a \triangleleft b) \cdots) \triangleleft b(N$ nested parentheses to the right of $b)$ for any $a, b \in X$. The associated group $\operatorname{As}(X)$ of $X$ is defined to be the group expressed by

$$
\left.\operatorname{As}(X):=\left\langle e_{x}\right| e_{x \triangleleft y}^{-1} e_{y}^{-1} e_{x} e_{y} \quad \text { for } x, y \in X\right\rangle .
$$

The group $\operatorname{As}(X)$ acts on $X$ by the formula $x \cdot e_{y}:=x \triangleleft y$ for $x, y \in X$. If the action is transitive, $X$ is said to be connected. If we have a homomorphism $\varepsilon_{X}: \operatorname{As}(X) \rightarrow \mathbb{Z}$ sending $e_{x}$ to 1 , we get an exact group extension

$$
0 \longrightarrow \operatorname{Ker}\left(\varepsilon_{X}\right) \stackrel{\iota}{\longrightarrow} \operatorname{As}(X) \stackrel{\varepsilon_{X}}{\longrightarrow} \mathbb{Z} \longrightarrow 0 .
$$

Next we introduce a subclass of quandles that we mainly investigate in this paper.

Definition 2.1 (Joyce [12, Section 4]) Fix a group $G$ and a group isomorphism $\rho: G \rightarrow G$. Equip $X=G$ with a quandle operation by setting

$$
g \triangleleft h:=\rho\left(g h^{-1}\right) h .
$$


Note that the quandle $(G, \rho)$ is of type $t_{X}$ if and only if $t_{X}$ is the smallest number for which $\rho^{t_{X}}=\mathrm{id}_{G}$ holds.

Although the $(G, \rho)$ only form a subclass of quandles, they include interesting examples:

Example 2.2 (Alexander quandle) Let $X=G$ be an abelian group. Denoting $\rho$ by $T$, we can regard $X$ as a $\mathbb{Z}\left[T, T^{-1}\right]$-module. Then the quandle operation is rewritten as

$$
x \triangleleft y:=T x+(1-T) y
$$

and $X$ is called an Alexander quandle. Given a finite field $\mathbb{F}_{q}$ and $\omega \in \mathbb{F}_{q}^{\times}$with $\omega \neq 1$, the quandle $X=\mathbb{F}_{q}[T] /(T-\omega)$ is called an Alexander quandle on $\mathbb{F}_{q}$ with $\omega$.

The type $t_{X}$ of $X$ equals the smallest $n$ satisfying $T^{n}=1$ in $X$. We can easily check that $X$ is connected if and only if $(1-T)$ is invertible.

Example 2.3 (Universal quandle covering) Given a connected quandle $X$, consider the kernel $G=\operatorname{Ker}\left(\varepsilon_{X}\right)$ in (4). Fix $a \in X$. Using a group homomorphism $\rho_{a}: \operatorname{Ker}\left(\varepsilon_{X}\right) \rightarrow \operatorname{Ker}\left(\varepsilon_{X}\right)$ defined by $\rho_{a}(g)=e_{a}^{-1} g e_{a}$, we have a quandle $\tilde{X}=$ $\left(\operatorname{Ker}\left(\varepsilon_{X}\right), \rho_{a}\right)$, called an extended quandle of $X$. We can easily check the independence of the choice of $a \in X$ up to quandle isomorphisms.

Considering the restriction of the action $X \curvearrowleft \operatorname{As}(X)$ to $\operatorname{Ker}\left(\varepsilon_{X}\right)$, the map $p_{X}: \tilde{X} \rightarrow X$ sending $g$ to $a \cdot g$ is known to be a quandle homomorphism (see [12, Theorem 4.1]), and is called a (universal quandle) covering [7]. It can easily be seen that if $X$ is of type $t_{X}$ and of finite order, then so is $\tilde{X}$. Furthermore, the quandle $\tilde{X}$ is connected [22, Lemma 6.8].

Finally, we briefly review the quandle complexes introduced in [3]. Let $X$ be a quandle. Let us construct a complex by considering the free $\mathbb{Z}$-module $C_{n}^{R}(X)$ spanned by $\left(x_{1}, \ldots, x_{n}\right) \in X^{n}$ and letting the boundary $\partial_{n}^{R}\left(x_{1}, \ldots, x_{n}\right) \in C_{n-1}^{R}(X)$ be

$$
\sum_{2 \leq i \leq n}(-1)^{i}\left(\left(x_{1}, \ldots, x_{i-1}, x_{i+1}, \ldots, x_{n}\right)-\left(x_{1} \triangleleft x_{i}, \ldots, x_{i-1} \triangleleft x_{i}, x_{i+1}, \ldots, x_{n}\right)\right) \text {. }
$$

The composite $\partial_{n-1}^{R} \circ \partial_{n}^{R}$ is zero. The pair $\left(C_{*}^{R}(X), \partial_{*}^{R}\right)$ is called a rack complex. Let $C_{n}^{D}(X)$ be a submodule of $C_{n}^{R}(X)$ generated by $n$-tuples $\left(x_{1}, \ldots, x_{n}\right)$ with $x_{i}=x_{i+1}$ for some $i \in\{1, \ldots, n-1\}$ if $n \geq 2$; otherwise, let $C_{1}^{D}(X)=0$. Since $\partial_{n}^{R}\left(C_{n}^{D}(X)\right) \subset C_{n-1}^{D}(X)$, we can define a complex $\left(C_{*}^{Q}(X), \partial_{*}\right)$ by the quotient $C_{n}^{R}(X) / C_{n}^{D}(X)$. The homology $H_{n}^{Q}(X)$ is called the quandle homology of $X$. Dually, we can define the cohomologies $H_{R}^{n}(X ; A)$ and $H_{Q}^{n}(X ; A)$ where $A$ is a commutative ring. 
However, the second term of the differential $\partial_{n}^{R}$ seems to be incomprehensible. In the next subsection, for quandles of the form $(G, \rho)$, we give another simple definition of $\partial_{n}^{R}$.

\subsection{A lift of Inoue-Kabaya chain map}

We now construct a chain map (7) with respect to a class of quandles in Definition 2.1. Our construction is a modification of the Inoue-Kabaya map [11, Section 3] (see the Remark below).

In this subsection, we often denote $\rho(x)$ by $x^{\rho}$ and $\rho^{n}(x)$ by $x^{n \rho}$, respectively.

For a quandle $X$ of the form $(G, \rho)$ in Definition 2.1, we will reformulate the rack complex $C_{n}^{R}(X)\left(\cong \mathbb{Z}\left\langle G^{n}\right\rangle\right)$ in non-homogeneous coordinates. To be specific, we consider a module isomorphism $\Upsilon: C_{n}^{R}(X) \cong C_{n}^{R}(X)$ derived from the bijection

$$
\Upsilon:\left(x_{1}, \ldots, x_{n}\right) \mapsto\left(x_{1} x_{2}^{-1}, x_{2} x_{3}^{-1}, \ldots, x_{n-1} x_{n}^{-1}, x_{n}\right),
$$

and we define another differential $\partial_{n}^{R_{G}}: C_{n}^{R}(X) \rightarrow C_{n-1}^{R}(X)$ to be the composite $-\Upsilon \circ \partial_{n}^{R} \circ \Upsilon^{-1}$. Then a direct calculation shows the following:

Lemma 2.4 For generators $\left(g_{1}, \ldots, g_{n}\right) \in C_{n}^{R}(X)$, the differential $\partial_{n}^{R_{G}}$ is

$$
\begin{aligned}
\partial_{n}^{R_{G}}\left(g_{1}, \ldots, g_{n}\right)= & \sum_{1 \leq i \leq n-1}(-1)^{i}\left(\left(g_{1}, \ldots, g_{i-1}, g_{i} g_{i+1}, g_{i+2}, \ldots, g_{n}\right)\right. \\
& \left.-\left(g_{1}^{\rho}, \ldots, g_{i-1}^{\rho}, g_{i}^{\rho} g_{i+1}, g_{i+2}, \ldots, g_{n}\right)\right) .
\end{aligned}
$$

When we discuss this $\partial_{n}^{R_{G}}$, we often denote the module $C_{n}^{R}(X)$ by $C_{n}^{R_{G}}(X)$. We also define a subcomplex $D_{n}(G)$ generated by $n$-tuples $\left(g_{1}, \ldots, g_{n}\right)$ such that $g_{i}=1$ for some $i \leq n-1$. We denote the quotient complex $C_{n}^{R_{G}}(G) / D_{n}(G)$ by $C_{n}^{Q_{G}}(X)$. Its homology $H_{n}^{Q_{G}}(X)$ is isomorphic to the quandle homology $H_{n}^{Q}(X)$ in Section 2.1. Next we give a brief review of the normalized chain complexes $C_{n}^{\mathrm{gr}}(G)$ of groups in non-homogeneous terms (see Brown [2]) as follows: Let $\bar{C}_{n}^{\mathrm{gr}}(G)$ denote the free $\mathbb{Z}$-module generated by $G^{n}$, and let its boundary map $\partial_{n}^{\mathrm{gr}}\left(g_{1}, \ldots, g_{n}\right) \in \bar{C}_{n-1}^{\mathrm{gr}}(G)$ be

$$
\begin{aligned}
\left(g_{2}, \ldots, g_{n}\right)+\sum_{1 \leq i \leq n-1}(-1)^{i}\left(g_{1}, \ldots, g_{i-1}, g_{i} g_{i+1}, g_{i+2}, \ldots, g_{n}\right) \\
+(-1)^{n}\left(g_{1}, \ldots, g_{n-1}\right) .
\end{aligned}
$$

We can easily check that $\partial_{n}^{\mathrm{gr}}\left(D_{n}(G)\right) \subset D_{n-1}(G)$ for the submodule $D_{n}(G)$ mentioned above. Let $C_{n}^{\mathrm{gr}}(G)$ denote the quotient complex of $\bar{C}_{n}^{\mathrm{gr}}(G)$ modulo $D_{n}(G)$. As is well known, the associated homology $H\left(C_{*}^{\mathrm{gr}}(G)\right)$ coincides with the usual group homology of $G$ (see [2, Section I.5]).

We now construct a chain map $\varphi_{n}$ from the complex $C_{n}^{R_{G}}(X)$ to $C_{n}^{\mathrm{gr}}(G)$. 
Definition 2.5 Assume that a quandle $X$ of the form $(G, \rho)$ is of type $t_{X}$. Take a set

$$
\mathcal{K}_{n}:=\left\{\left(k_{1}, \ldots, k_{n}\right) \in \mathbb{Z}^{n} \mid 0 \leq k_{i-1}-k_{i} \leq 1,0 \leq k_{n} \leq t_{X}-1\right\}
$$

of order $t_{X} 2^{n-1}$. We define a homomorphism $\varphi_{n}: C_{n}^{R_{G}}(X) \rightarrow C_{n}^{\mathrm{gr}}(G)$ by setting

$$
\varphi_{n}\left(g_{1}, g_{2}, \ldots, g_{n}\right)=\sum_{\left(k_{1}, \ldots, k_{n}\right) \in \mathcal{K}_{n}}(-1)^{k_{1}-k_{n}}\left(g_{1}^{k_{1} \rho}, g_{2}^{k_{2} \rho}, \ldots, g_{n}^{k_{n} \rho}\right) \in C_{n}^{\mathrm{gr}}(G) .
$$

For example, when $n=3, \varphi_{3}(x, y, z)$ is written as

$$
\begin{aligned}
\sum_{0 \leq i \leq t_{X}-1}\left(x^{i \rho}, y^{i \rho}, z^{i \rho}\right)-( & \left.x^{(i+1) \rho}, y^{i \rho}, z^{i \rho}\right) \\
& -\left(x^{(i+1) \rho}, y^{(i+1) \rho}, z^{i \rho}\right)+\left(x^{(i+2) \rho}, y^{(i+1) \rho}, z^{i \rho}\right) .
\end{aligned}
$$

Proposition 2.6 Let $X$ be a quandle of the form $(G, \rho)$. If $X$ is of type $t_{X}<\infty$, then the homomorphism $\varphi_{n}: C_{n}^{R_{G}}(X) \rightarrow C_{n}^{\mathrm{gr}}(G)$ is a chain map. To be specific, $\partial_{n}^{\mathrm{gr}} \circ \varphi_{n}=\varphi_{n-1} \circ \partial_{n}^{R_{G}}$.

Furthermore, the image of $D_{n}(G)$ under this map is zero. The $\varphi_{n}$ induce a chain map from the quotient $C_{n}^{Q_{G}}(X)$ to $C_{n}^{\mathrm{gr}}(G)$ and a homomorphism $H_{n}^{Q_{G}}(X) \rightarrow H_{n}^{\mathrm{gr}}(G)$.

Proof Since the identity $\partial_{n}^{\mathrm{gr}} \circ \varphi_{n}=\varphi_{n-1} \circ \partial_{n}^{R_{G}}$ is proven similarly those in [11, Lemma 3.1] or [21, Appendix], we defer the details to the Appendix. It is easy to check the latter part directly by using the definitions.

We realize an easy construction of quandle cocycles from group cocycles:

Corollary 2.7 Let a quandle $X=(G, \rho)$ be of type $t_{X}$. For any normalized group $n$-cocycle $\kappa$ of $G$, the pullback $\varphi_{n}^{*}(\kappa)$ is a quandle $n$-cocycle.

Remark We roughly compare our map $\varphi_{n}$ with a chain map $\varphi_{\mathrm{IK}}$ introduced by Inoue and Kabaya [11]. For any quandle $Q$, they constructed a complex $C_{n}^{\Delta}(Q)$ from a simplicial object and formulated the map $\varphi_{\mathrm{IK}}: C_{n}^{R}(Q) \rightarrow C_{n}^{\Delta}(Q)$ in its homogeneous coordinate system (see [11, Section 3] for details).

To see this in greater detail, we define a module $C_{n}^{\mathrm{gr}}(G)_{\mathbb{Z}}$ as the quotient of $C_{n}^{\mathrm{gr}}(G)$ modulo the relation $\left(g_{1}, \ldots, g_{n}\right)=\left(\rho\left(g_{1}\right), \ldots, \rho\left(g_{n}\right)\right)$, called the $\mathbb{Z}$-coinvariant of $C_{n}^{\mathrm{gr}}(G)$. Let $\pi_{\rho}$ denote the projection $C_{n}^{\mathrm{gr}}(G) \rightarrow C_{n}^{\mathrm{gr}}(G)_{\mathbb{Z}}$. We can see that if $Q$ is a connected quandle of the form $(G, \rho)$, then the above complex $C_{n}^{\Delta}(Q)$ is isomorphic to the coinvariant $C_{n}^{\mathrm{gr}}(G)_{\mathbb{Z}}$; further, we can check the equality $t_{X} \cdot \varphi_{\mathrm{IK}}=\pi_{\rho} \circ \varphi_{n}$. In summary, our map $\varphi_{n}$ is of a lift of the Inoue-Kabaya map $\varphi_{\mathrm{IK}}$ in connected cases and is relatively simple. Therefore we fix some notation: 
Definition 2.8 Let $\Phi_{n}$ be the composite chain map $\pi_{\rho} \circ \varphi_{n}: C_{n}^{Q_{G}}(X) \rightarrow C_{n}^{\mathrm{gr}}(G)_{\mathbb{Z}}$, that is,

$$
\Phi_{n}: C_{n}^{Q_{G}}(X) \stackrel{\varphi_{n}}{\longrightarrow} C_{n}^{\mathrm{gr}}(G) \stackrel{\pi_{\rho}}{\longrightarrow} C_{n}^{\mathrm{gr}}(G)_{\mathbb{Z}} .
$$

Incidentally, we prepare a "reduced map" of the $\Phi_{n}$, which is used temporarily in Theorem 2.15. Consider a homomorphism $\mathcal{P}: C_{n}^{R_{G}}(X) \rightarrow C_{n-1}^{R_{G}}(X)$ derived from a map $X^{n} \rightarrow X^{n-1}$ sending $\left(x_{1}, \ldots, x_{n}\right)$ to $\left(x_{1}, \ldots, x_{n-1}\right)$. We discuss the composite $\Phi_{n-1} \circ \mathcal{P}:$

Proposition 2.9 Let $X$ be a quandle $(G, \rho)$ of type $t_{X}$. The composite

$$
\Phi_{n-1} \circ \mathcal{P}: C_{n}^{R_{G}}(X) \rightarrow C_{n-1}^{\mathrm{gr}}(G)_{\mathbb{Z}}
$$

is a chain map. Furthermore, it induces a chain map from the quotient $C_{n}^{Q_{G}}(X)$ to $C_{n-1}^{\mathrm{gr}}(G)_{\mathbb{Z}}$.

Proof From the definitions, first note that

$$
\left(\partial_{n-1}^{R_{G}} \circ \mathcal{P}-\mathcal{P} \circ \partial_{n}^{R_{G}}\right)\left(g_{1}, \ldots, g_{n}\right)=(-1)^{n}\left(\left(g_{1}, \ldots, g_{n-2}\right)-\left(g_{1}^{\rho}, \ldots, g_{n-2}^{\rho}\right)\right) .
$$

The map $\Phi_{n-1}$ sends this element to zero by the definitions of $\mathcal{K}_{n}$ and $\varphi_{n}$. Here, we use $\rho^{t_{X}}(g)=g$ for any $g \in G$. Since $\varphi_{n}$ is a chain map (Proposition 2.6), so is the composite.

\subsection{Results on the chain map $\Phi_{3}$}

We now study the chain map $\Phi_{n}$ with $n=3$ (see Theorems 2.10, 2.13).

First we study the maps $\Phi_{n}$ with respect to extended quandles in Example 2.3.

Theorem 2.10 Let $X$ be a connected quandle of type $t_{X}$, and let $\tilde{X}=\left(\operatorname{Ker}\left(\varepsilon_{X}\right), \rho_{a}\right)$ be the extended quandle in Example 2.3. Let $\widetilde{\Phi}_{n}$ denote the chain map in Definition 2.8. Assume that the $H_{3}^{\mathrm{gr}}(\operatorname{As}(X))$ is finitely generated (eg $X$ is of finite order). Then the induced map

$$
\left(\widetilde{\Phi}_{3}\right)_{*}: H_{3}^{Q}(\tilde{X}) \rightarrow H_{3}^{\mathrm{gr}}\left(\operatorname{Ker}\left(\varepsilon_{X}\right)\right)_{\mathbb{Z}}
$$

is an isomorphism modulo $t_{X}$-torsion.

Remark 2.11 We can compare this theorem with [22, Theorem 3.18], which states the existence of an isomorphism $H_{3}^{Q}(\tilde{X}) \cong H_{3}^{\mathrm{gr}}(\operatorname{As}(X))$ modulo $t_{X}$. Later, in Lemma 3.4, we obtain a canonical isomorphism $H_{3}^{\mathrm{gr}}(\operatorname{As}(X)) \cong H_{3}^{\mathrm{gr}}\left(\operatorname{Ker}\left(\varepsilon_{X}\right)\right)_{\mathbb{Z}}$ modulo $t_{X}$. Thus this theorem says that the chain map $\left(\widetilde{\Phi}_{3}\right)_{*}$ gives an explicit presentation of this isomorphism. 
Next, as a special case, we focus on the Alexander quandles on $\mathbb{F}_{q}$ in Example 2.2. Using the maps $\Phi_{n}$, we will characterize the third quandle cohomology from group homologies. Identifying $X=\mathbb{F}_{q}$ with $\left(\mathbb{Z}_{p}\right)^{h}$ as an additive group, let $\rho: \mathbb{F}_{q} \rightarrow \mathbb{F}_{q}$ denote the multiplication by $\omega$. Then we have a chain map $\Phi_{n}^{*}: C_{\mathrm{gr}}^{n}\left(\left(\mathbb{Z}_{p}\right)^{h}\right)^{\mathbb{Z}} \rightarrow C_{Q}^{n}(X)$ and will later show the following:

Proposition 2.12 Let $X$ be an Alexander quandle on $\mathbb{F}_{q}$ with $\omega$ as in Example 2.2. Let $q=p^{h}$. The induced map $\Phi_{3}^{*}: H_{\mathrm{gr}}^{3}\left(\left(\mathbb{Z}_{p}\right)^{h} ; \mathbb{F}_{q}\right)^{\mathbb{Z}} \rightarrow H_{Q}^{3}\left(X ; \mathbb{F}_{q}\right)$ is injective.

Furthermore, if $H_{2}^{Q}(X)$ vanishes, then this $\Phi_{3}^{*}$ is an isomorphism.

In general, this $\Phi_{3}^{*}$ is not surjective. To get around the obstruction $H_{2}^{Q}(X)$, we consider the chain map $\widetilde{\Phi}_{n}: C_{n}^{Q}(\tilde{X}) \rightarrow C_{n}^{\mathrm{gr}}\left(\operatorname{Ker}\left(\varepsilon_{X}\right)\right)_{\mathbb{Z}}$ with respect to the extended quandle (Example 2.3). As seen in Section 4, the abelianization of $\operatorname{Ker}\left(\varepsilon_{X}\right)$ is given by Proj: $\operatorname{Ker}\left(\varepsilon_{X}\right) \rightarrow\left(\mathbb{Z}_{p}\right)^{h}$ (as a set-theoretic projection). Then we obtain a commutative diagram:

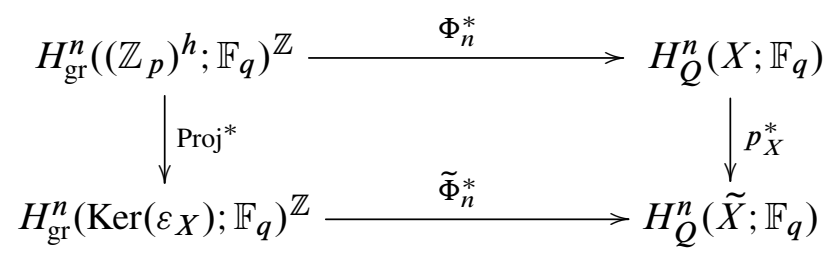

We remark that when $n=3$, the bottom map $\widetilde{\Phi}_{3}^{*}$ is an isomorphism by Theorem 2.10. Let $\operatorname{res}\left(\widetilde{\Phi}_{3}^{*}\right)$ denote the isomorphism restricted to the cokernel Coker(Proj $\left.{ }^{*}\right)$. In addition, we take the chain map $\Phi_{n-1} \circ \mathcal{P}: C_{n}^{Q_{G}}(X) \rightarrow C_{n-1}^{\mathrm{gr}}(G)_{\mathbb{Z}}$ in Proposition 2.9.

To summarize these homomorphisms, we characterize the third quandle cohomology of $X$ :

Theorem 2.13 Let $X$ be an Alexander quandle on $\mathbb{F}_{q}$. Let $q=p^{h}$ be odd. Then there is a section $\mathfrak{s}: H_{Q}^{3}\left(\tilde{X} ; \mathbb{F}_{q}\right) \rightarrow H_{Q}^{3}\left(X ; \mathbb{F}_{q}\right)$ of $p_{X}^{*}$ such that the following homomorphism is an isomorphism:

$$
\begin{aligned}
\left(\Phi_{2} \circ \mathcal{P}\right)^{*} \oplus \Phi_{3}^{*} \oplus\left(\mathfrak{s} \circ \operatorname{res}\left(\tilde{\Phi}_{3}^{*}\right)\right): & \\
& H_{\mathrm{gr}}^{2}\left(\left(\mathbb{Z}_{p}\right)^{h} ; \mathbb{F}_{q}\right)^{\mathbb{Z}} \oplus H_{\mathrm{gr}}^{3}\left(\left(\mathbb{Z}_{p}\right)^{h} ; \mathbb{F}_{q}\right)^{\mathbb{Z}} \oplus \operatorname{Coker}\left(\operatorname{Proj}^{*}\right) \rightarrow H_{Q}^{3}\left(X ; \mathbb{F}_{q}\right) .
\end{aligned}
$$

The proof will appear in Section 4. Although the paper [22, Theorem 3.16] showed the existence of an isomorphism

$$
H_{Q}^{3}\left(X ; \mathbb{F}_{q}\right) \cong H_{3}^{\mathrm{gr}}(\operatorname{As}(X)) \oplus\left(H_{2}^{Q}(X) \wedge H_{2}^{Q}(X)\right),
$$


this theorem gives an explicit formulation of the isomorphism in some sense, and implies clearly that all the Mochizuki 3-cocycles are derived from group 3-cocycles of $\left(\mathbb{Z}_{p}\right)^{h}$ and $\operatorname{Ker}\left(\varepsilon_{X}\right)$ via the chain map $\Phi_{n}$.

Incidentally, at a higher degree, we now observe that the induced map $\left(\varphi_{n}\right)_{*}: H_{n}^{Q}(X) \rightarrow$ $H_{n}^{\mathrm{gr}}(G)$ is far from injective and surjective.

Example 2.14 To see this, we let $q=p$ and examine the chain map $\varphi_{n}$ with respect to an Alexander quandle $X$ on $\mathbb{F}_{p}$ in Example 2.3. There we see that $\operatorname{Ker}\left(\varepsilon_{X}\right) \cong \mathbb{Z}_{p}$ (cf (20)); hence $H_{\mathrm{gr}}^{n}\left(\mathbb{Z}_{p} ; \mathbb{F}_{p}\right) \cong \mathbb{F}_{p}$ for any $n \in \mathbb{N}$. Nosaka [20] showed that the integral quandle homology $H_{n}^{Q}(X)$ is $\left(\mathbb{Z}_{p}\right)^{b_{n}}$, where $b_{n} \in \mathbb{Z}$ is determined by the recurrence formula

$b_{n+2 t}=b_{n}+b_{n+1}+b_{n+2}, \quad b_{1}=b_{2}=\cdots=b_{2 t-2}=0 \quad$ and $\quad b_{2 t-1}=b_{2 t}=1$, and $t>0$ is the minimal number satisfying $\omega^{t}=1$. In conclusion, since the $b_{n}$ grow exponentially, the map $\left(\varphi_{n}\right)_{*}$ is not bijective.

\subsection{Shadow cocycle invariant and Dijkgraaf-Witten invariant}

We address a topological relation between the shadow cocycle invariant [4] and the Dijkgraaf-Witten invariant [6]. We will review both the invariants and state Theorem 2.15.

First, to describe the former invariant, we review $X$-colorings. Given a quandle $X$, an $X$-coloring of an oriented link diagram $D$ is a map $\mathcal{C}:\{\operatorname{arcs}$ of $D\} \rightarrow X$ satisfying the condition on the left-hand side of Figure 1 at each crossing of $D$. Let $\operatorname{Col}_{X}(D)$ denote the set of all $X$-colorings of $D$. Note that two diagrams $D_{1}$ and $D_{2}$ related by Reidemeister moves admit a 1:1 correspondence $\operatorname{Col}_{X}\left(D_{1}\right) \leftrightarrow \operatorname{Col}_{X}\left(D_{2}\right)$; see [3; 4] for details.

We define a shadow coloring to be a pair consisting of an $X$-coloring $\mathcal{C}$ and a map $\lambda$ from the complementary regions of $D$ to $X$ such that, if regions $R$ and $R^{\prime}$ are separated by an arc $\alpha$ as shown on the right-hand side of Figure 1, the equality $\lambda(R) \triangleleft \mathcal{C}(\alpha)=\lambda\left(R^{\prime}\right)$ holds. Let $\overline{\mathrm{Col}}_{X}(D)$ denote the set of shadow colorings of $D$. Given an $X$-coloring $\mathcal{C}$, we put $x_{0} \in X$ on the region containing a point at infinity. Then, by the rules in Figure 1, the colors of other regions are uniquely determined, and they ensure a shadow coloring $\mathcal{S}$ denoted by $\left(\mathcal{C} ; x_{0}\right)$. Thus, we obtain a bijection $\operatorname{Col}_{X}(D) \times X \simeq \overline{\operatorname{Col}}_{X}(D)$ sending $\left(\mathcal{C}, x_{0}\right)$ to $\mathcal{S}=\left(\mathcal{C} ; x_{0}\right)$.

We briefly formulate (shadow) quandle cocycle invariants [4]. Let $D$ be a diagram of a link $L$, and let $\mathcal{S} \in \overline{\mathrm{Col}}_{X}(D)$ be a shadow coloring. For a crossing $\tau$ shown in 

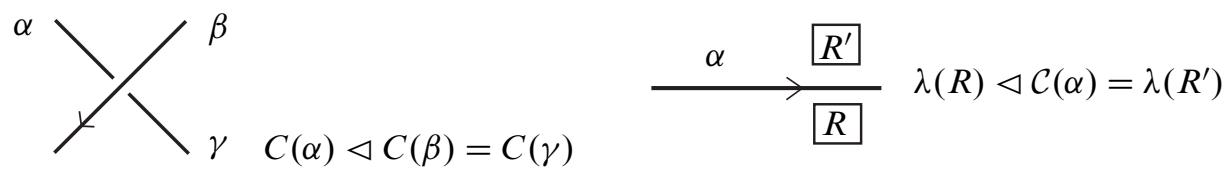

Figure 1: Coloring conditions at each crossing and around arcs. In this paper, we describe orientations of links as normal orientations.

Figure 2, we define a weight of $\tau$ to be $\epsilon_{\tau}(x, y, z) \in C_{3}^{Q}(X ; \mathbb{Z})$, where $\epsilon_{\tau} \in\{ \pm 1\}$ is the sign of $\tau$ according to Figure 2. Then, the fundamental class of $\mathcal{S}$ is defined to be $\sum_{\tau} \epsilon_{\tau}(x, y, z) \in C_{3}^{Q}(X ; \mathbb{Z})$, and it is known to be a 3-cycle. We denote the homology class by $[\mathcal{S}]$. For a quandle 3 -cocycle $\psi \in C_{Q}^{3}(X ; A)$, we consider the pairing $\langle\psi,[\mathcal{S}]\rangle \in A$. If $X$ is of finite order, the formal sum $I_{\psi}(L):=\sum_{\mathcal{S} \in \overline{\mathrm{Col}}_{X}(D)} 1_{\mathbb{Z}}\{\langle\psi,[\mathcal{S}]\rangle\}$ in the group ring $\mathbb{Z}[A]$ is called the quandle cocycle invariant of $L$, where the symbol $1_{\mathbb{Z}}\{a\} \in \mathbb{Z}[A]$ denotes the generator corresponding to $a \in A$. By construction, in order to calculate the invariant concretely, it is important to find explicit formulas for quandle 3 -cocycles.
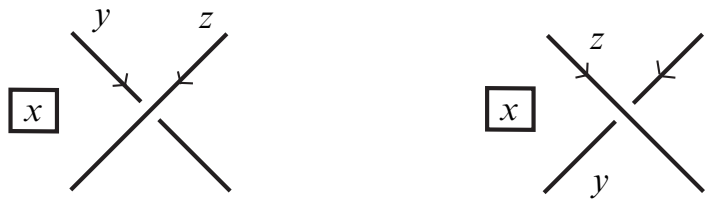

Figure 2: Positive and negative crossings with $X$-colors

On the other hand, we will briefly formulate a Dijkgraaf-Witten invariant below (10). For a link $L$, let $\widehat{C}_{L}^{m}$ denote the $m$-fold cyclic covering space of $S^{3}$ branched over $L$. Note that $\mathbb{Z}$ canonically acts on the space $\widehat{C}_{L}^{m}$ by covering transformations. According to [22], when $X$ is connected and of type $t$, for an $X$-coloring of $L$, we can construct a $\mathbb{Z}$-equivariant homomorphism $\Gamma_{\mathcal{C}}: \pi_{1}\left(\widehat{C}_{L}^{t}\right) \rightarrow \operatorname{Ker}\left(\varepsilon_{X}\right)$, where $\mathbb{Z}$ acts on $\operatorname{Ker}\left(\varepsilon_{X}\right)$ via the homomorphism $\rho_{a}$ in Example 2.3; see Section 3.1 for the definition of $\Gamma_{\mathcal{C}}$. In summary, given a link-diagram $D$, we have a map

$$
\Gamma_{\bullet}: \operatorname{Col}_{X}(D) \rightarrow \operatorname{Hom}_{\mathrm{gr}}^{\mathbb{Z}}\left(\pi_{1}\left(\hat{C}_{L}^{t}\right), \operatorname{Ker}\left(\varepsilon_{X}\right)\right),
$$

where the right-hand side is the set of the $\mathbb{Z}$-equivariant group homomorphisms $\pi_{1}\left(\widehat{C}_{L}^{t}\right) \rightarrow \operatorname{Ker}\left(\varepsilon_{X}\right)$. Furthermore, consider the pushforward of the fundamental class $\left[\widehat{C}_{L}^{t}\right] \in H_{3}\left(\hat{C}_{L}^{t}\right)$ via the induced map $\left(\Gamma_{\mathcal{C}}\right)_{*}: H_{3}\left(\hat{C}_{L}^{t}\right) \rightarrow H_{3}^{\mathrm{gr}}\left(\operatorname{Ker}\left(\varepsilon_{X}\right)\right)$. Using this, with respect to a $\mathbb{Z}$-invariant 3 -cocycle $\kappa$ of $\operatorname{Ker}\left(\varepsilon_{X}\right)$, we define $a \mathbb{Z}$-equivariant 
part of the Dijkgraaf-Witten invariant of $\widehat{C}_{L}^{t}$ by the formula

$$
\operatorname{DW}_{\kappa}^{\mathbb{Z}}\left(\widehat{C}_{L}^{t}\right)=\sum_{\mathcal{C} \in \operatorname{Col}_{X}(D)}\left\langle\kappa,\left(\Gamma_{\mathcal{C}}\right)_{*}\left(\left[\widehat{C}_{L}^{t}\right]\right)\right\rangle \in \mathbb{Z}[A] .
$$

We remark that this invariant depends on the link $L$, and not on only the topological type of the cyclic branched coverings space $\widehat{C}_{L}^{t}$ with the $\mathbb{Z}$-action on their fundamental group. Actually, in general, the map (9) is not surjective.

Next we state that with respect to a connected quandle that satisfies a certain assumption, the two invariants explained above are equivalent (see Section 3 for the proof).

Theorem 2.15 Let $X$ be a finite connected quandle of type $t_{X}$. Assume that an abelian group $A$ contains no $t_{X}$-torsion, and the induced map $p_{X}^{*}: H_{Q}^{3}(X ; A) \rightarrow H_{Q}^{3}(\tilde{X} ; A)$ is surjective. Then any $\mathbb{Z}$-invariant 3-cocycle $\kappa$ of $\operatorname{Ker}\left(\varepsilon_{X}\right)$ admits a quandle 3-cocycle $\psi$ of $X$, which ensures the equality

$$
I_{\psi}(L)=|X| \cdot \operatorname{DW}_{\kappa}^{\mathbb{Z}}\left(\hat{C}_{L}^{t_{X}}\right) \in \mathbb{Z}[A] .
$$

Conversely, given a quandle 3-cocycle $\psi$ of $X$, there is a $\mathbb{Z}$-invariant group 3-cocycle $\kappa$ of $\operatorname{Ker}\left(\varepsilon_{X}\right)$ for which the equality holds.

Remark 2.16 As seen in in Section 3.2, for some quandles, we can obtain the quandle cocycle $\psi$ in Theorem 2.15 concretely from a group 3-cocycle $\kappa$. For instance, if $p_{X}: \tilde{X} \rightarrow X$ is an isomorphism, then $\psi$ is given by $\varphi_{3}^{*}(\kappa)$. As another example, for Alexander quandles on $\mathbb{F}_{q}$, the relations between $\psi$ and $\kappa$ are given by explicit formulas (see Section 4.2 in detail).

As mentioned in the introduction, the homotopical equivalence of the two invariants was implied in [22]. However, this theorem gives the equivalence explicitly in the cohomological viewpoint and hence it serves to compute some parts of the DijkgraafWitten invariants $\mathrm{DW}_{\kappa}\left(\widehat{C}_{L}^{t_{X}}\right)$ via the quandle cocycle invariants $I_{\psi}(L)$.

To conclude, under the assumption on the $p_{X}^{*}$, the invariant $\mathrm{DW}_{\kappa}^{\mathbb{Z}}\left(\widehat{C}_{L}^{t}\right)$ constructed from any $\mathbb{Z}$-invariant 3-cocycle $\kappa$ of $\operatorname{Ker}\left(\varepsilon_{X}\right)$ can be computed from the quandle cocycle invariants via link-diagrams. Fortunately, there are some quandles satisfying the assumption of the surjectivity of $p_{X}^{*}$ : for example, connected Alexander quandles $X$ such that the order $|X|$ is odd or $t_{X}$ is even [22, Lemma 9.15], and "symplectic quandles $X$ over $\mathbb{F}_{q}$ " with $g=1$ [22, Section 3.3].

In contrast, other quandles do not satisfy the assumption. In fact, with respect to symplectic quandles $X$ with $g>1$ in a stable range, which are of type $p$, it has been 
shown [22, Section 3] that

$$
H_{3}^{\mathrm{gr}}\left(\operatorname{Ker}\left(\varepsilon_{X}\right)\right) \cong H_{3}^{\mathrm{gr}}\left(\operatorname{Sp}\left(2 g ; \mathbb{F}_{q}\right)\right) \cong \mathbb{Z} / q^{2}-1 \text { and } H_{3}^{Q}(X) \cong 0 .
$$

Hence the invariant $\mathrm{DW}_{\kappa}^{\mathbb{Z}}\left(\hat{C}_{L}^{t_{X}}\right)$ can not always be interpreted from shadow cocycle invariants.

\section{Proofs of Theorems 2.10 and 2.15}

We will prove Theorems 2.10 and 2.15 . The idea of the proofs is, roughly speaking, to reduce of the chain map $\varphi_{3}$ to two homomorphisms from a certain group $\Pi_{2}(\tilde{X})$. As was shown in [22], the homomorphisms are isomorphisms in some cases, which play roles in the proofs. Thus Section 3.1 reviews the group $\Pi_{2}(X)$ and the two homomorphisms. In Section 3.2, we prove the theorems using the homomorphisms and a key lemma. In Section 3.3, we give a proof of the key lemma. Readers who are interested in only Theorem 2.13 may skip to Section 4.

\subsection{Review of two homomorphisms $\Delta_{X, x_{0}}$ and $\Theta_{X}$}

We construct the two homomorphisms that appear in (11) and (12) below. For this, we start by reviewing the group $\Pi_{2}(X)$ defined in [8;9]. Consider the set of all $X$-colorings of all link-diagrams. We define $\Pi_{2}(X)$ to be the quotient set subject to Reidemeister moves and concordance relations illustrated in Figure 3. Disjoint unions of $X$-colorings make $\Pi_{2}(X)$ into an abelian group. For a connected quandle $X$ of finite order, the group $\Pi_{2}(X)$ has been well-studied (see Theorem 3.1 below).
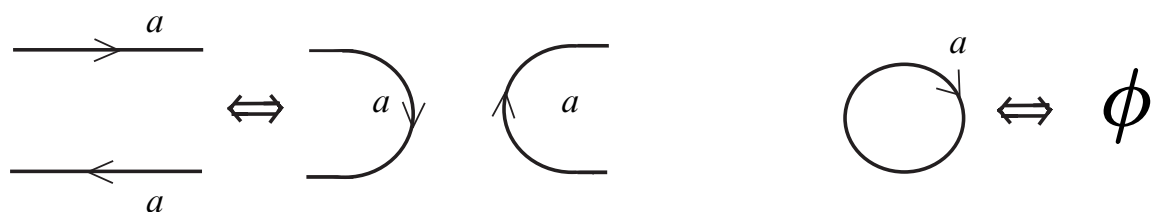

Figure 3: The concordance relations

Next we will explain the first homomorphism (11). Recall from Section 2.4 that given an $X$-coloring $\mathcal{C}$ and $x_{0} \in X$, we can construct a shadow coloring of the form $\left(\mathcal{C} ; x_{0}\right)$ and the fundamental class $\left[\left(\mathcal{C} ; x_{0}\right)\right]$ contained in $H_{3}^{Q}(X)$. We easily see that if two $X$-colorings $\mathcal{C}, \mathcal{C}^{\prime}$ are related by Reidemeister moves and concordance relations, then the associated classes $\left[\left(\mathcal{C} ; x_{0}\right)\right],\left[\left(\mathcal{C}^{\prime} ; x_{0}\right)\right]$ are equal in $H_{3}^{Q}(X)$ by definition. Hence we obtain a homomorphism

$$
\Delta_{X, x_{0}}: \Pi_{2}(X) \rightarrow H_{3}^{Q}(X), \quad \mathcal{C} \mapsto\left[\left(\mathcal{C} ; x_{0}\right)\right] .
$$


We will now explain the second $\Theta_{X}$, which appears in (12). To this end, we first examine the fundamental group of the $t$-fold cyclic branched covering $\widehat{C}_{L}^{t}$. Given a link-diagram $D$ of $L$, let $\gamma_{0}, \ldots, \gamma_{n}$ be the arcs of $D$. Consider the Wirtinger presentation of $\pi_{1}\left(S^{3} \backslash L\right)$ generated by $\gamma_{0}, \ldots, \gamma_{n}$. For $s \in \mathbb{Z}$, we take a copy $\gamma_{i, s}$ of the arc $\gamma_{i}$. Then by the Reidemeister-Schreier method (see [13, Section 3] for the details), the group $\pi_{1}\left(\hat{C}_{L}^{t}\right)$ can be presented by

generators $\gamma_{i, s}(0 \leq i \leq n, s \in \mathbb{Z})$

relations $\quad \gamma_{k, s}=\gamma_{j, s-1}^{-1} \gamma_{i, s-1} \gamma_{j, s} \quad$ for each crossing in the figure below $\gamma_{i, s}=\gamma_{i, s+t}, \quad \gamma_{0, s}=1$
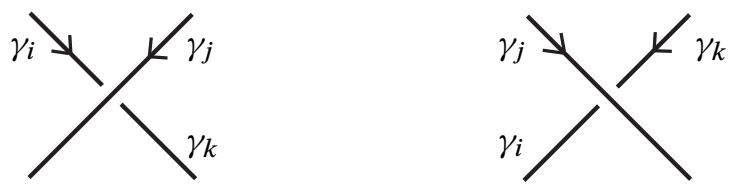

Let $X$ be a connected quandle of type $t$. Given an $X$-coloring $\mathcal{C} \in \operatorname{Col}_{X}(D)$, we denote the color on the arc $\gamma_{i}$ by $x_{i} \in X$. Define a group homomorphism $\Gamma_{\mathcal{C}}: \pi_{1}\left(\hat{C}_{L}^{t}\right) \rightarrow$ $\operatorname{Ker}\left(\varepsilon_{X}\right)$ by setting

$$
\Gamma_{\mathcal{C}}\left(\gamma_{i, s}\right):=e_{x_{0}}^{s-1} e_{x_{i}} e_{x_{0}}^{-s}
$$

(this is well-defined; see [22, Section 4]). Furthermore, considering the fundamental class, $\left[\hat{C}_{L}^{t}\right]$, in $H_{3}\left(\hat{C}_{L}^{t}\right)$, it gives a (group) homology class in $H_{3}^{\mathrm{gr}}\left(\pi_{1}\left(\hat{C}_{L}^{t}\right)\right)$. We often denote the pushforward of this homology class under $\left(\Gamma_{\mathcal{C}}\right)_{*}$ by $\left(\Gamma_{\mathcal{C}}\right)_{*}\left(\left[\widehat{C}_{L}^{t}\right]\right) \in$ $H_{3}^{\mathrm{gr}}\left(\operatorname{Ker}\left(\varepsilon_{X}\right)\right)$ in abuse of notation. We thus obtain a map

$$
\theta_{X, D}: \operatorname{Col}_{X}(D) \rightarrow H_{3}^{\mathrm{gr}}\left(\operatorname{Ker}\left(\varepsilon_{X}\right)\right), \quad \mathcal{C} \mapsto\left(\Gamma_{\mathcal{C}}\right)_{*}\left(\left[\widehat{C}_{L}^{t}\right]\right)
$$

As is shown [22], if two $X$-colorings $\mathcal{C}, \mathcal{C}^{\prime}$ can be related by Reidemeister moves and concordance relations, then $\theta_{X, D}(\mathcal{C})=\theta_{X, D^{\prime}}\left(\mathcal{C}^{\prime}\right)$. Therefore the maps $\theta_{X, D}$ with respect to all diagrams $D$ yield a homomorphism

$$
\Theta_{X}: \Pi_{2}(X) \rightarrow H_{3}^{\mathrm{gr}}\left(\operatorname{Ker}\left(\varepsilon_{X}\right)\right) .
$$

This $\Theta_{X}$ plays an important role in the study of the group $\Pi_{2}(X)$ up to $t$-torsion.

Theorem 3.1 [22, Theorems 3.4 and 3.18 $]^{1} \quad$ Let $X$ be a connected quandle of type $t_{X}$. Recall the inclusion $\iota: \operatorname{Ker}\left(\varepsilon_{X}\right) \rightarrow \operatorname{As}(X)$ in (4). If the homology $H_{3}^{\mathrm{gr}}(\operatorname{As}(X))$ is finitely generated, then $\iota_{*} \circ \Theta_{X}: \Pi_{2}(X) \rightarrow H_{3}^{\mathrm{gr}}(\mathrm{As}(X))$ is a split surjection modulo $t_{X}$-torsion, whose kernel is isomorphic to $H_{2}^{Q}(X)$ modulo $t_{X}$-torsion.

Furthermore, the induced map $\left(p_{X}\right)_{*}: \Pi_{2}(\tilde{X}) \rightarrow \Pi_{2}(X)$ is a split injection modulo $t_{X}$-torsion, and this cokernel is isomorphic to the kernel of the composite $\iota_{*} \circ \Theta_{X}$.

\footnotetext{
${ }^{1}$ In [22], the maps $\Theta_{X}$ and $\iota_{*} \circ \Theta_{X}$ were denoted by $\Theta_{\Pi \Omega}, \Theta_{X}$, respectively.
} 


\subsection{A key lemma and proofs of Theorems 2.10 and 2.15}

For the proofs, we state a key lemma. We here fix terminology: A (shadow) $\tilde{X}$-coloring of $D$ is said to be based if an arc $\gamma_{0}$ of $D$ is colored by the identity $1_{\operatorname{Ker}\left(\varepsilon_{X}\right)} \in \tilde{X}$.

Lemma $3.2\left(\operatorname{cf}[13 \text {, Theorem 9.1] }]^{2}\right.$ Let $X$ be a connected quandle of type $t_{X}<\infty$, and let $p_{X}: \tilde{X} \rightarrow X$ be the projection in Example 2.3. Take the chain isomorphism $\Upsilon: C_{3}^{R}(\tilde{X}) \rightarrow C_{3}^{R_{G}}(\tilde{X})$ described in (6). Let $\mathcal{S} \in \overline{\operatorname{Col}} \tilde{X}(D)$ be a based shadow coloring. Let $\tilde{\mathcal{C}} \in \operatorname{Col} \tilde{X}(D)$ be the restriction of $\mathcal{S} \in \overline{\operatorname{Col}}_{\tilde{X}}(D)$. Then

$$
\Theta_{X}\left(\left[p_{X}(\tilde{\mathcal{C}})\right]\right)=\varphi_{3} \circ \Upsilon([\mathcal{S}]) \in H_{3}^{\mathrm{gr}}\left(\operatorname{Ker}\left(\varepsilon_{X}\right)\right) .
$$

Before proving the lemma, we will complete the proofs of Theorems 2.10 and 2.15.

Proof of Theorem 2.10 As mentioned in Remark 2.11, there is an isomorphism $H_{3}^{Q}(\tilde{X}) \cong H_{3}^{\mathrm{gr}}(\operatorname{As}(X))$ up to $t_{X}$-torsion, as finitely generated $\mathbb{Z}$-modules. Hence, in order to prove that the map $\left(\widetilde{\Phi}_{3}\right)_{*}$ is an isomorphism, it is enough to show it is surjective. To this end, we set the composite of the three homomorphisms mentioned above:

$$
\Pi_{2}(\tilde{X}) \stackrel{\left(p_{X}\right)_{*}}{\longrightarrow} \Pi_{2}(X) \stackrel{\Theta_{X}}{\longrightarrow} H_{3}^{\mathrm{gr}}\left(\operatorname{Ker}\left(\varepsilon_{X}\right)\right) \stackrel{\iota_{*}}{\longrightarrow} H_{3}^{\mathrm{gr}}(\operatorname{As}(X)) .
$$

It follows from Theorem 3.1 that this composite is an isomorphism up to $t_{X}$-torsion. Note that Lemma 3.4 below ensures the existence of an isomorphism

$$
\xi: H_{3}^{\mathrm{gr}}(\operatorname{As}(X)) \rightarrow H_{3}^{\mathrm{gr}}\left(\operatorname{Ker}\left(\varepsilon_{X}\right)\right)_{\mathbb{Z}}
$$

such that $\xi \circ \iota_{*}=t_{X} \cdot\left(\pi_{\rho}\right)_{*}$, where $\pi_{\rho}$ is the projection $C_{3}^{\mathrm{gr}}\left(\operatorname{Ker}\left(\varepsilon_{X}\right)\right) \rightarrow C_{3}^{\mathrm{gr}}\left(\operatorname{Ker}\left(\varepsilon_{X}\right)\right)_{\mathbb{Z}}$ explained in Section 2.2. Hence, the following composite is an isomorphism up to $t_{X}$-torsion as well:

$$
\left(\pi_{\rho}\right)_{*} \circ \Theta_{X} \circ\left(p_{X}\right)_{*}: \Pi_{2}(\tilde{X}) \longrightarrow H_{3}^{\mathrm{gr}}\left(\operatorname{Ker}\left(\varepsilon_{X}\right)\right)_{\mathbb{Z}} .
$$

Therefore, for any 3 -cycle $\mathcal{K} \in H_{3}^{\mathrm{gr}}\left(\operatorname{Ker}\left(\varepsilon_{X}\right)\right)_{\mathbb{Z}}$ that is annihilated by $t_{X}$, we can choose some based $\tilde{X}$-coloring $\widetilde{\mathcal{C}}$ such that $\mathcal{K}=\left(\pi_{\rho}\right)_{*} \circ \Theta_{X} \circ\left(p_{X}\right)_{*}([\widetilde{\mathcal{C}}])$. We here set a shadow coloring $\mathcal{S}$ of the form $\left(\widetilde{\mathcal{C}} ; 1_{\tilde{X}}\right)$. Then by the key Lemma 3.2 , we notice the equalities

$$
\widetilde{\Phi}_{3}(\Upsilon([\mathcal{S}]))=\left(\pi_{\rho}\right)_{*} \circ\left(\varphi_{3} \circ \Upsilon\right)([\mathcal{S}])=\left(\pi_{\rho}\right)_{*} \circ \Theta_{X}\left(p_{X}([\widetilde{\mathcal{C}}])\right)=\mathcal{K} .
$$

Since $\mathcal{K}$ is arbitrary, we obtain the surjectivity of $\widetilde{\Phi}_{3}$ as required.

\footnotetext{
${ }^{2}$ Kabaya [13] showed a similar statement under a certain strong condition of quandles. However, as seen in the proofs of Theorems 2.10 and 2.15, in order to verify a relation to the Dijkgraaf-Witten invariant, we may deal with only the extended quandle $\tilde{X}$ in Example 2.3, which may not satisfy the strong condition. The point is that Kabaya's condition (16) is strong and does not hold in most cases.
} 
Proof of Theorem 2.15 We first construct two homomorphisms (13), (14) below. Let $X$ be a finite connected quandle of type $t_{X}$. Given a $\mathbb{Z}$-invariant group 3-cocycle $\kappa$, consider a composite homomorphism from $\Pi_{2}(X)$ :

$$
\Pi_{2}(X) \stackrel{\Theta_{X}}{\longrightarrow} H_{3}^{\mathrm{gr}}\left(\operatorname{Ker}\left(\varepsilon_{X}\right)\right) \stackrel{\langle\kappa, \bullet\rangle}{\longrightarrow} A .
$$

On the other hand, by the assumption that

$$
p_{X}^{*}: H_{Q}^{3}(X ; A) \rightarrow H_{Q}^{3}(\tilde{X} ; A)
$$

is surjective, we can choose a quandle cocycle $\psi \in H_{Q}^{3}(X ; A)$ such that $p_{X}^{*}(\psi)=$ $\left(\varphi_{3} \circ \Upsilon\right)^{*}(\kappa)$. We then set a composite homomorphism

$$
\Pi_{2}(X) \stackrel{\left[\bullet ; x_{0}\right]}{\longrightarrow} H_{3}^{Q}(X) \stackrel{\langle\psi, \bullet\rangle}{\longrightarrow} A .
$$

We remark that this kernel contains the kernel of $\Theta_{X}$ by Theorem 3.1, since $A$ contains no $t_{X}$-torsion by assumption.

Next we claim the equivalence of the two maps (13) and (14). For this, we choose $\tilde{X}-$ colorings $\tilde{\mathcal{C}}_{1}, \ldots, \tilde{\mathcal{C}}_{n}$ that generate $\Pi_{2}(\tilde{X})$; here we may assume that these colorings are based by Lemma 3.3 below. Notice that, by Theorem 3.1, the group $\Pi_{2}(X)$ is generated by the kernel $\operatorname{Ker}\left(\iota_{*} \circ \Theta_{X}\right)$ and the elements $p_{X}\left(\tilde{\mathcal{C}}_{1}\right), \ldots, p_{X}\left(\tilde{\mathcal{C}}_{n}\right)$. Therefore, the claimed equivalence results from the equalities

$$
\begin{aligned}
\left\langle\kappa, \Theta_{X}\left(p_{X}\left(\widetilde{\mathcal{C}_{i}}\right)\right\rangle\right. & =\left\langle\kappa, \varphi_{3} \circ \Upsilon\left(\left[\left(\tilde{\mathcal{C}}_{i} ; \tilde{x}_{0}\right)\right]\right)\right\rangle \\
& \left.=\left\langle p_{X}^{*}(\psi),\left[\left(\tilde{\mathcal{C}}_{i} ; \tilde{x}_{0}\right)\right]\right\rangle=\left\langle\psi,\left[p_{X}\left(\tilde{\mathcal{C}}_{i}\right) ; p_{X}\left(\tilde{x}_{0}\right)\right)\right]\right\rangle,
\end{aligned}
$$

where the first equality is obtained from Lemma 3.2.

We further show the equivalence of the two invariants as stated in Theorem 2.15. By definition, these invariants are reformulated as

$$
\begin{aligned}
\operatorname{DW}_{\kappa}^{\mathbb{Z}}\left(\hat{C}_{L}^{t_{X}}\right) & =\sum_{\mathcal{C} \in \operatorname{Col}_{X}(D)} 1_{\mathbb{Z}}\left\{\left\langle\kappa, \Theta_{X}(\mathcal{C})\right\rangle\right\}, \\
I_{\psi}(L) & =\sum_{x \in X} \sum_{\mathcal{C} \in \operatorname{Col}_{X}(D)} 1_{\mathbb{Z}}\{\langle\psi,[\mathcal{C} ; x])\} \in \mathbb{Z}[A] .
\end{aligned}
$$

We know that $I_{\psi}(L)=|X| \sum_{\mathcal{C} \in \operatorname{Col}_{X}(D)} 1_{\mathbb{Z}}\left\{\left\langle\psi,\left[\mathcal{C} ; x_{0}\right]\right)\right\}$ for any $x_{0} \in X[11$, Theorem 4.3]. In conclusion, since the homomorphisms (13), (14) are equal as claimed above, so are the two invariants.

Finally, to prove the latter part of Theorem 2.15 , recall that the map $\widetilde{\Phi}_{3}$ is an isomorphism after tensoring by $A$ (Theorem 2.10). So, given a quandle 3 -cocycle $\psi$, 
we define a $\mathbb{Z}$-invariant group 3-cocycle $\kappa$ of $\operatorname{Ker}\left(\varepsilon_{X}\right)$ to be $\left(\Upsilon \circ \widetilde{\Phi}_{3}^{*}\right)^{-1}\left(p_{X}^{*}(\psi)\right)$. Hence, by a similar argument as above, we have the desired equality

$$
I_{\psi}(L)=|X| \cdot \mathrm{DW}_{\kappa}^{\mathbb{Z}}\left(\hat{C}_{L}^{t_{X}}\right) .
$$

\subsection{Proof of the key lemma}

We will prove Lemma 3.2 as a modification of [13, Theorem 9.1].

For this purpose, we review descriptions in [13, Section 4] to formulate concretely the orientation class $\left[\widehat{C}_{L}^{t}\right] \in H_{3}\left(\hat{C}_{L}^{t} ; \mathbb{Z}\right)$ of the branched covering space $\hat{C}_{L}^{t}$. Let $c_{0}, \ldots, c_{n}$ be the crossings of the diagram $D$. For each crossing $c_{i}$, we can construct 4 tetrahedra $T_{i}^{(u)} \subset S^{3}$ with $1 \leq u \leq 4$, and further decompose the 3 -sphere $S^{3}$ into these $4(n+1)$ tetrahedra. Furthermore, set up $4 t$ tetrahedra $T_{i, s}^{(u)}$ that correspond to lifts of $T_{i}^{(u)}$, where $0 \leq s<t$ and $1 \leq u \leq 4$, and consider the action of $\mathbb{Z} / t$ on the set $\left\{T_{i, s}^{(u)}\right\}_{i, s, u}$ defined by $\left(T_{i, 0}^{(u)}\right) * s=T_{i, s}^{(u)}$. Let us fix the orderings of the vertices of $T_{i, s}^{(u)}$ following Kabaya in [13, Figure 8]. There, Kabaya glued each of the tetrahedra $T_{i, s}^{(u)}$ along appropriate boundary triangles of other tetrahedra (see [13, Figures 7 and 14]). Then he showed that the resulting union $\bigcup_{i, s, u} T_{i, s}^{(u)}$ is homeomorphic to the branched covering space $\widehat{C}_{L}^{t}$, that the homeomorphism is compatible with the orientations and the $\mathbb{Z}-$ actions, and that, in particular, the formal sum $\sum_{i, s} \epsilon_{i}\left(T_{i, s}^{(1)}-T_{i, s}^{(2)}-T_{i, s}^{(3)}+T_{i, s}^{(4)}\right)$ represents the orientation class $\left[\hat{C}_{L}^{t}\right]$, where $\epsilon_{i} \in\{ \pm 1\}$ is the sign of the crossing $c_{i}$.

Moreover, he examined the pushforward $f_{*}\left(\left[\widehat{C}_{L}^{t}\right]\right)$ with respect to a homomorphism $f: \pi_{1}\left(\hat{C}_{L}^{t}\right) \rightarrow G$ as below. Let us set up some situations to give a labeling of the tetrahedron $T_{i, s}^{(u)}$ by a group $G$. Let us take the generators $\gamma_{i, s} \in \pi_{1}\left(\widehat{C}_{L}^{t}\right)$ in Section 3.1, and assume we have the identities

$$
\begin{aligned}
& f\left(\gamma_{k, s}\right)=f\left(\gamma_{j, s-1}\right)^{-1} f\left(\gamma_{i, s}\right) f\left(\gamma_{j, s}\right) \quad(i, j, k \text { as in Figure } 2, s=1, \ldots, t), \\
& f\left(\gamma_{0,1}\right)=f\left(\gamma_{0,2}\right)=\cdots=f\left(\gamma_{0, t}\right)=1_{G} .
\end{aligned}
$$

Also, consider a map $\mathcal{L}:\left\{T_{i, s}^{(u)}\right\}_{i, s, u} \rightarrow G^{3}$ satisfying the conditions

$$
\mathcal{L}\left(T_{i, s}^{(1)}\right) \cdot f\left(\gamma_{i, s}\right)=\mathcal{L}\left(T_{i, s+1}^{(3)}\right), \quad \mathcal{L}\left(T_{i, s}^{(2)}\right) \cdot f\left(\gamma_{i, s}\right)=\mathcal{L}\left(T_{i, s+1}^{(4)}\right) \in G^{3},
$$

where the symbol - means the diagonal multiplication. We assume that if a point $x \in \widehat{C}_{L}^{t}$ is contained as a vertex in two tetrahedra, then the two images of $x$ under $\mathcal{L}$ are equal in $G$.

Condider the constant map $\mathcal{I}:\left\{T_{i, s}^{(u)}\right\}_{i, s, u} \rightarrow G$ that sends all elements to the identity of $G$. We here regard the product $\mathcal{I} \times \mathcal{L}$ as a labeling of vertices in $T_{i, s}^{(u)}$ according to the vertices of the ordering. As is seen in $[6 ; 23]$, we have a (simplicial) map from 
the 1-skeleton of the union $\bigcup_{i, s, u} T_{i, s}^{(u)}$ to the Eilenberg-MacLane space $K(G, 1)$; hence we have a group homomorphism from $\pi_{1}\left(\left[\hat{C}_{L}^{t}\right]\right)$ to $G$. Then, as a result of [13, Section 4], the condition (16) implies that this homomorphism coincides with the original $f$. In what followed, the pushforward $f_{*}\left(\left[\hat{C}_{L}^{t}\right]\right)$ was shown to be represented by the formula

$$
\Upsilon\left(\sum_{0 \leq i \leq n} \sum_{0 \leq s<t} \mathcal{L}\left(\epsilon_{i}\left(T_{i, s}^{(1)}-T_{i, s}^{(2)}-T_{i, s}^{(3)}+T_{i, s}^{(4)}\right)\right)\right) \in C_{3}^{\mathrm{gr}}(G),
$$

where $\Upsilon: C_{n}^{\mathrm{gr}}(G) \rightarrow C_{n}^{\mathrm{gr}}(G)$ is the isomorphism defined in (6).

Proof of Lemma 3.2 We will prove the lemma by expressing the left-hand side $\Theta_{X}\left(p_{X}(\widetilde{\mathcal{C}})\right)$ in detail. We later denote the $X$-coloring $p_{X}(\widetilde{\mathcal{C}})$ by $\mathcal{C}$ for short.

Starting from the based shadow $\tilde{X}$-coloring $\mathcal{S}$, we will define a labeling $\mathcal{L}$ compatible with the group homomorphism $\Gamma_{\mathcal{C}}: \pi_{1}\left(\widehat{C}_{L}^{t}\right) \rightarrow \operatorname{Ker}\left(\varepsilon_{X}\right)$ in (9). For each crossing $c_{i}$, let $(g, h, k) \in \tilde{X}^{3}=\operatorname{Ker}\left(\varepsilon_{X}\right)^{3}$ be the weight of $c_{i}$. Using the quandle structure on $\tilde{X}$, we then define a map $\mathcal{L}:\left\{T_{i, s}^{(u)}\right\}_{s, i, u} \rightarrow \operatorname{Ker}\left(\varepsilon_{X}\right)^{3}=\tilde{X}^{3}$ by

$$
\begin{array}{ll}
\mathcal{L}\left(T_{i, s}^{(1)}\right):=\left(g_{s-1}, h_{s-1}, k_{s-1}\right), \quad & \mathcal{L}\left(T_{i, s}^{(2)}\right):=\left(g_{s-1} \triangleleft h_{s-1}, h_{s-1}, k_{s-1}\right), \\
\mathcal{L}\left(T_{i, s}^{(3)}\right):=\left(g_{s} \triangleleft k_{s}, h_{s} \triangleleft k_{s}, k_{s}\right), & \mathcal{L}\left(T_{i, s}^{(4)}\right):=\left(\left(g_{s} \triangleleft h_{s}\right) \triangleleft k_{s}, h_{s} \triangleleft k_{s}, k_{s}\right),
\end{array}
$$

where we temporarily use notation $g_{s}:=e_{a}^{s} g e_{a}^{-s} \in \operatorname{Ker}\left(\varepsilon_{X}\right)$ for short.

We will verify the equalities (16) for this $\mathcal{L}$ in order to apply $\Gamma_{\mathcal{C}}$ to $f$. From the definition of the action $X \curvearrowleft \operatorname{As}(X)$, we notice an equality $e_{p_{X}(k)}=e_{a \cdot k}=k^{-1} e_{a} k \in$ $\operatorname{As}(X)$ for any $k \in \tilde{X}$. In addition, we note $\left(p_{X}\right)_{*}\left(\mathcal{S}\left(\gamma_{0}\right)\right)=p_{X}\left(1_{\tilde{X}}\right)=a \in X$ since the $\mathcal{S}$ is based by assumption. Hence, using the generator $\gamma_{i, s} \in \pi_{1}\left(\hat{C}_{L}^{t}\right)$, we have

$$
\Gamma_{\mathcal{C}}\left(\gamma_{i, s}\right)=\left(e_{a}\right)^{s-1} e_{a \cdot \mathcal{S}\left(\gamma_{i}\right)} e_{a}^{-s}=\left(e_{a}\right)^{s-1} e_{p_{X}(k)} e_{a}^{-s}=e_{a}^{s-1} k^{-1} e_{a} k e_{a}^{-s} .
$$

Using (18) we see that for any $b \in X$, we have the identity

$$
\left(e_{a}^{s-1} b e_{a}^{1-s}\right) \cdot \Gamma_{\mathcal{C}}\left(\gamma_{i, s}\right)=e_{a}^{s}(b \triangleleft k) e_{a}^{-s} \in \operatorname{Ker}\left(\varepsilon_{X}\right) .
$$

Plugging the three cases $b=g, b=h$ or $b=g \triangleleft h$ into this identity establishes condition (16).

Hence the labeling $\mathcal{L}$ yields the homomorphism $\Gamma_{\mathcal{C}}: \pi_{1}\left(\widehat{C}_{L}^{t}\right) \rightarrow \operatorname{As}(X)$. Actually, the homomorphism $f=\Gamma_{\mathcal{C}}$ satisfies (15) by definition, and it is not hard to see that the labels $\mathcal{L}$ of any two vertexes that are identified in the union $\bigcup_{i, s, u} T_{i, s}^{(u)}$ are exactly equal, where we follow the description of the tetrahedra by Kabaya [13, Section 3, 4]. 
Finally, we discuss the pushforward of the orientation class $\left(\Gamma_{\mathcal{C}}\right)_{*}\left(\left[\widehat{C}_{L}^{t}\right]\right) \in C_{3}^{\mathrm{gr}}\left(\operatorname{Ker}\left(\varepsilon_{X}\right)\right)$. We first check that, for any $x, y, z \in \tilde{X}=\operatorname{Ker}\left(\varepsilon_{X}\right)$, we have $-\Upsilon^{-1} \circ \varphi_{3} \circ \Upsilon(x, y, z)$

$$
\begin{aligned}
=\sum_{1 \leq s \leq t}\left(x_{s}, y_{s}, z_{s}\right)-\left(x_{s} \triangleleft y_{s}, y_{s}, z_{S}\right) & -\left(x_{s} \triangleleft z_{S}, y_{s} \triangleleft z_{S}, z_{S}\right) \\
& +\left(\left(x_{s} \triangleleft y_{S}\right) \triangleleft z_{S}, y_{S} \triangleleft z_{S}, z_{S}\right) .
\end{aligned}
$$

This verification is easily obtained by recalling the definitions of $\varphi_{3}$ in Definition 2.5 and $\Upsilon$ in (6). Hence, comparing with the map $\mathcal{L}$, we immediately have the equality

$$
-\Upsilon^{-1} \circ \varphi_{3} \circ \Upsilon([\mathcal{S}])=\sum_{i} \sum_{s} \mathcal{L}\left(\epsilon_{i}\left(T_{i, s}^{(1)}-T_{i, s}^{(2)}-T_{i, s}^{(3)}+T_{i, s}^{(4)}\right)\right) \in C_{3}^{\mathrm{gr}}\left(\operatorname{Ker}\left(\varepsilon_{X}\right)\right)
$$

exactly. Notice that the right-hand side is the push-forward $\Upsilon^{-1}\left(\left(\Gamma_{\mathcal{C}}\right)_{*}\left(\left[\widehat{C}_{L}^{t}\right]\right)\right)$ by (17). Hence, from the definition of $\Theta_{X}$, we conclude the desired equality in Lemma 3.2.

We now provide proofs of the two lemmas which we used above.

Lemma 3.3 Let $X$ be a connected quandle. Any element in $\Pi_{2}(\tilde{X})$ is represented by a class of some based $\tilde{X}$-coloring.

Proof Let $\mathcal{C}$ be an $\tilde{X}$-coloring representing the element and $h \in \tilde{X}$ be the $X$-color of the arc $\gamma_{0}$. Since the extended quandle $\tilde{X}$ is also connected [22, Lemma 9.15], we have $g_{1}, \ldots, g_{n} \in \tilde{X}$ such that $\left(\cdots\left(h \triangleleft g_{1}\right) \triangleleft \cdots\right) \triangleleft g_{n}=1 \tilde{X}$. Then, by considering the following picture, we can change $\mathcal{C}$ to another $\tilde{X}$-coloring $\mathcal{C}^{\prime}$ of $D$ such that the arc $\gamma_{0}$ is colored by $h \triangleleft g_{1}$ and that $[\mathcal{C}]=\left[\mathcal{C}^{\prime}\right] \in \Pi_{2}(\tilde{X})$.

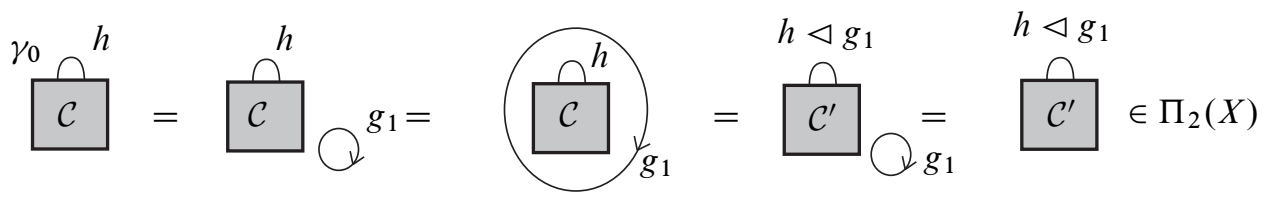

Here the first and forth equalities are obtained from the concordance relation, and in the second (resp. third) equality, the loop colored by $g_{1}$ passes under (resp. over) all the $\operatorname{arcs}$ of $D$. Note that here we only use Reidemeister moves. Hence, iterating this process, we have a based $\tilde{X}$-coloring $\mathcal{C}^{(n)}$ of $D$ such that $[\mathcal{C}]=\left[\mathcal{C}^{(n)}\right] \in \Pi_{2}(\tilde{X})$.

Lemma 3.4 Let $X$ be a connected quandle of type $t_{X}$. Let $\iota: \operatorname{Ker}\left(\varepsilon_{X}\right) \rightarrow \operatorname{As}(X)$ be the inclusion (4), and $\pi_{\rho}: C_{3}^{\mathrm{gr}}\left(\operatorname{Ker}\left(\varepsilon_{X}\right)\right) \rightarrow C_{3}^{\mathrm{gr}}\left(\operatorname{Ker}\left(\varepsilon_{X}\right)\right)_{\mathbb{Z}}$ be the projection. Then there is an isomorphism $\xi: H_{3}^{\mathrm{gr}}(\operatorname{As}(X)) \rightarrow H_{3}^{\mathrm{gr}}\left(\operatorname{Ker}\left(\varepsilon_{X}\right)\right)_{\mathbb{Z}}$ modulo $t_{X}$-torsion such that $\xi \circ l_{*}=t_{X} \cdot\left(\pi_{\rho}\right)_{*}$. 
Proof Fix $x \in X$ and consider the subgroup $\left\langle e_{x}^{n t_{X}}\right\rangle_{n \in \mathbb{Z}}$ of $\operatorname{As}(X)$, which is contained in the center (see [22, Lemma 4.1]). Define the quotient $Q_{X}:=\operatorname{As}(X) /\left\langle e_{x}^{n t_{X}}\right\rangle_{n \in \mathbb{Z}}$. By the Lyndon-Hochschild spectral sequence, the projection $P$ induces an isomorphism $P_{*}: H_{3}^{\mathrm{gr}}(\operatorname{As}(X)) \cong H_{3}^{\mathrm{gr}}\left(Q_{X}\right)$ up to $t_{X}$-torsion, since $H_{2}^{\mathrm{gr}}(\operatorname{As}(X))$ is annihilated by $t_{X}$ [22, Corollary 6.4]. Furthermore, noting the group extension $\operatorname{Ker}\left(\varepsilon_{X}\right) \rightarrow Q_{X} \rightarrow \mathbb{Z} / t_{X}$, the transfer gives an isomorphism $\mathcal{T}: H_{3}^{\mathrm{gr}}\left(Q_{X}\right) \rightarrow H_{3}^{\mathrm{gr}}\left(\operatorname{Ker}\left(\varepsilon_{X}\right)\right)_{\mathbb{Z}}$ modulo $t_{X}$; see [2, Section III.10]. Hence, denoting $\mathcal{T} \circ P_{*}$ by $\xi$, we have the equality $\xi \circ \iota_{*}=t_{X} \cdot\left(\pi_{\rho}\right)_{*}$ by construction.

\section{Proofs of Proposition 2.12 and Theorem 2.13}

The outline of the proofs of Proposition 2.12 and Theorem 2.13 is as follows. A basis for the third cohomology of an Alexander quandle over $\mathbb{F}_{q}$ was found by Mochizuki [19], which we review in Section 4.1. We will see that it is enough to show that the map in (8) is surjective. So we will construct group 3-cocycles of $\operatorname{As}(X)$ as preimages of the basis via the chain maps $\Phi_{3}$ and $\widetilde{\Phi}_{3}$ (see Section 4.2).

To implement this outline, we start by reviewing a simple presentation of $\operatorname{As}(X)$, where $X$ of a connected Alexander quandle, according to Clauwens [5]. Consider a tensor product $X \otimes X$ over $\mathbb{Z}$ to be an abelian group. Define a homomorphism $\mu_{X}: X \otimes X \rightarrow X \otimes X$ by

$$
\mu_{X}(x \otimes y)=x \otimes y-T y \otimes x .
$$

We equip a product $\mathbb{Z} \times X \times \operatorname{Coker}\left(\mu_{X}\right)$ with a group operation given by

$$
(n, a, \kappa) \cdot(m, b, v)=\left(n+m, T^{m} a+b, \kappa+v+\left[T^{m} a \otimes b\right]\right) .
$$

Then a homomorphism $\operatorname{As}(X) \rightarrow \mathbb{Z} \times X \times \operatorname{Coker}\left(\mu_{X}\right)$ sending the generators $e_{x}$ to $(1, x, 0)$ is an isomorphism [5, Theorem 1]. Then we easily see that the lower central series of $\operatorname{As}(X)$ are then described as

$$
\operatorname{As}(X) \supset X \times \operatorname{Coker}\left(\mu_{X}\right) \supset \operatorname{Coker}\left(\mu_{X}\right) \supset 0 .
$$

In particular, the kernel $\operatorname{Ker}\left(\varepsilon_{X}\right)$ in (4) is a subgroup of the set $X \times \operatorname{Coker}\left(\mu_{X}\right)$. Incidentally, there is an isomorphism $H_{2}^{Q}(X) \cong \operatorname{Coker}\left(\mu_{X}\right)$ [5]. Note that the action of $\rho_{a}$ in Example 2.3 is reformulated as $\rho_{a}(0, x, \alpha)=(0, T x, \alpha)$.

Notation Denote by $G_{X}$ a subgroup of $X \times \operatorname{Coker}\left(\mu_{X}\right)$. In this section, we let $X$ be an Alexander quandle on $\mathbb{F}_{q}$ with $\omega \in \mathbb{F}_{q}$. Let $X$ be of type $t_{X}$. That is, $t_{X}$ is the minimal number satisfying $\omega^{t_{X}}=1$. Note that $t_{X}$ is coprime to $q$ since $\omega^{q-1}=1$. 


\subsection{Review of Mochizuki 3-cocycles}

We will review Mochizuki 2- and 3-cocycles of $X=\mathbb{F}_{q}$. We here regard polynomials in the ring $\mathbb{F}_{q}\left[U_{1}, \ldots, U_{n}\right]$ as functions from $X^{n}$ to $\mathbb{F}_{q}$, and as being in the complex $C_{Q_{G}}^{n}\left(X ; \mathbb{F}_{q}\right)$ in Section 2.2.

Theorem 4.1 [19, Lemma 3.7] The following set is a basis of $H_{Q}^{2}\left(X ; \mathbb{F}_{q}\right)$ :

$$
\left\{U_{1}^{q_{1}} U_{2}^{q_{2}} \mid \omega^{q_{1}+q_{2}}=1,1 \leq q_{1}<q_{2}<q \text { and } q_{i} \text { is a power of } p\right\} .
$$

Next, we describe all the quandle 3-cocycles of $X$. For this, recall the following three polynomials over $\mathbb{F}_{q}[19$, Section 2.2]:

$$
\begin{aligned}
\chi\left(U_{j}, U_{j+1}\right) & :=\sum_{1 \leq i \leq p-1}(-1)^{i-1} i^{-1} U_{j}^{p-i} U_{j+1}^{i} \\
& =\left(\left(U_{j}+U_{j+1}\right)^{p}-U_{j}^{p}-U_{j+1}^{p}\right) / p, \\
E_{0}(a \cdot p, b) & :=\left(\chi\left(\omega U_{1}, U_{2}\right)-\chi\left(U_{1}, U_{2}\right)\right)^{a} \cdot U_{3}^{b}, \\
E_{1}(a, b \cdot p) & :=U_{1}^{a} \cdot\left(\chi\left(U_{2}, U_{3}\right)-\chi\left(\omega^{-1} \cdot U_{2}, U_{3}\right)\right)^{b} .
\end{aligned}
$$

Define a set $I_{q, \omega}^{+}$consisting of the polynomials under some conditions:

$$
\begin{aligned}
& I_{q, \omega}^{+}:=\left\{E_{0}\left(q_{1} \cdot p, q_{2}\right) \mid \omega^{p \cdot q_{1}+q_{2}}=1, q_{1}<q_{2}\right\} \\
& \\
& \cup\left\{E_{1}\left(q_{1}, q_{2} \cdot p\right) \mid \omega^{q_{1}+p \cdot q_{2}}=1, q_{1} \leq q_{2}\right\} \\
& \cup\left\{U_{1}^{q_{1}} U_{2}^{q_{2}} U_{3}^{q_{3}} \mid \omega^{q_{1}+q_{2}+q_{3}}=1, q_{1}<q_{2}<q_{3}\right\} .
\end{aligned}
$$

Here the symbols $q_{i}$ range over powers of $p$ with $q_{i}<q$.

We review polynomials denoted by $\Gamma\left(q_{1}, q_{2}, q_{3}, q_{4}\right)$. For this, we define a set $\mathcal{Q}_{q, \omega} \subset$ $\mathbb{Z}^{4}$ consisting of quadruples $\left(q_{1}, q_{2}, q_{3}, q_{4}\right)$ such that:

- $q_{2} \leq q_{3}, q_{1}<q_{3}, q_{2}<q_{4}$, and $\omega^{q_{1}+q_{3}}=\omega^{q_{2}+q_{4}}=1$. Here, if $p=2$, we omit $q_{2}=q_{3}$.

- One of the following holds:

Case $1 \omega^{q_{1}+q_{2}}=1$.

Case $2 \omega^{q_{1}+q_{2}} \neq 1$ and $q_{3}>q_{4}$.

Case $3(p \neq 2), \omega^{q_{1}+q_{2}} \neq 1$ and $q_{3}=q_{4}$.

Case $4(p \neq 2), \omega^{q_{1}+q_{2}} \neq 1, q_{2} \leq q_{1}<q_{3}<q_{4}, \omega^{q_{1}}=\omega^{q_{2}}$.

Case $5(p=2), \omega^{q_{1}+q_{2}} \neq 1, q_{2}<q_{1}<q_{3}<q_{4}, \omega^{q_{1}}=\omega^{q_{2}}$. 
We call an element of the set $\mathcal{Q}_{q, \omega}$ a Mochizuki quadruple. For $\left(q_{1}, q_{2}, q_{3}, q_{4}\right) \in \mathcal{Q}_{q, \omega}$, in each case the associated polynomial $\Gamma\left(q_{1}, q_{2}, q_{3}, q_{4}\right)$ is defined as follows: ${ }^{3}$

Case $1 \Gamma\left(q_{1}, q_{2}, q_{3}, q_{4}\right):=U_{1}^{q_{1}} U_{2}^{q_{2}+q_{3}} U_{3}^{q_{4}}$.

Case $2 \Gamma\left(q_{1}, q_{2}, q_{3}, q_{4}\right):=U_{1}^{q_{1}} U_{2}^{q_{2}+q_{3}} U_{3}^{q_{4}}-U_{1}^{q_{2}} U_{2}^{q_{1}+q_{4}} U_{3}^{q_{3}}$ $-\left(\omega^{q_{2}}-1\right)^{-1}\left(1-\omega^{q_{1}+q_{2}}\right)\left(U_{1}^{q_{1}} U_{2}^{q_{2}} U_{3}^{q_{3}+q_{4}}-U_{1}^{q_{1}+q_{2}} U_{2}^{q_{4}} U_{3}^{q_{3}}\right)$.

Case $3 \quad \Gamma\left(q_{1}, q_{2}, q_{3}, q_{4}\right):=U_{1}^{q_{1}} U_{2}^{q_{3}+q_{4}} U_{3}^{q_{2}}$.

Cases 4, $5 \Gamma\left(q_{1}, q_{2}, q_{3}, q_{4}\right):=U_{1}^{q_{3}} U_{2}^{q_{1}+q_{2}} U_{3}^{q_{4}}$.

Remark 4.2 The 3-cocycle in Case 3 (resp. 4 and 5) is formulated as that in Case 1 after changing the indices $(1,2,3,4)$ to $(1,3,4,2)$ (resp. to $(3,1,2,4))$.

Theorem 4.3 [19] The following set composed of quandle 3-cocycles gives a basis of the third cohomology $H_{Q}^{3}\left(X ; \mathbb{F}_{q}\right)$. Here $q_{i}$ means a power of $p$ with $q_{i}<q$.

$I_{q, \omega}^{+} \cup\left\{\Gamma\left(q_{1}, q_{2}, q_{3}, q_{4}\right) \mid\left(q_{1}, q_{2}, q_{3}, q_{4}\right) \in \mathcal{Q}_{q, \omega}\right\} \cup\left\{U_{1}^{q_{1}} U_{2}^{q_{2}} \mid \omega^{q_{1}+q_{2}}=1, q_{1}<q_{2}\right\}$.

Remark Unfortunately the original statement and his proof of this theorem contained slight errors, which have been corrected by Mandemaker [17].

\subsection{Proofs of Proposition 2.12 and Theorem 2.13}

First, to prove Proposition 2.12, we prepare a lemma for a study of the quandle 3cocycles in (22), using the notation $\chi$ from (21):

Lemma 4.4 Let us identify $G=\left(\mathbb{Z}_{p}\right)^{h}$ with $\mathbb{F}_{q}$ as an additive group. Then the second group cohomology $H_{\mathrm{gr}}^{2}\left(G ; \mathbb{F}_{q}\right) \cong\left(\mathbb{F}_{q}\right)^{h(h+1) / 2}$ is generated by the group 2-cocycles

$$
\left\{U_{1}^{q_{1}} U_{2}^{q_{2}}, \chi\left(U_{1}, U_{2}\right)^{q_{3}} \mid 1 \leq q_{1}<q_{2}<q, 1 \leq q_{3}<q \text {, where } q_{i} \text { is a power of } p\right\} .
$$

Furthermore, $H_{\mathrm{gr}}^{3}\left(G ; \mathbb{F}_{q}\right) \cong \mathbb{F}_{q}{ }^{h(h+1)(h+2) / 6}$ is spanned by the 3 -cocycles

$\left\{U_{1}^{q_{1}} U_{2}^{q_{2}} U_{3}^{q_{3}} \mid q_{1}<q_{2}<q_{3}\right\} \cup\left\{\chi\left(U_{1}, U_{2}\right)^{q_{1}} \cdot U_{3}^{q_{2}} \mid q_{1}<q_{2}\right\}$

$$
\cup\left\{U_{1}^{q_{1}} \cdot \chi\left(U_{2}, U_{3}\right)^{q_{2}} \mid q_{1} \leq q_{2}\right\},
$$

where $q_{1}, q_{2}, q_{3}$ run over powers of $p$ with $1 \leq q_{j}<q$. Regarding the multiplication of $\omega \in \mathbb{F}_{q}$ as an action of $\mathbb{Z}$ on $\mathbb{F}_{q}$, the $\mathbb{Z}$-invariant parts $H_{\mathrm{gr}}^{i}\left(G ; \mathbb{F}_{q}\right)^{\mathbb{Z}}$ are generated by the above polynomials of degree $d$ satisfying $\omega^{d}=1$, where $i=2,3$.

\footnotetext{
${ }^{3}$ In Cases 3, 4 and 5, we change the forms of $\Gamma\left(q_{1}, q_{2}, q_{3}, q_{4}\right)$ in [19]; however, our $\Gamma$ are cohomologous to the original ones.
} 
Proof In the proof, we rely on the well-known graded ring isomorphism

$$
H_{\mathrm{gr}}^{*}\left(G ; \mathbb{F}_{q}\right) \cong \bigwedge\left(\alpha_{1}, \ldots, \alpha_{h}\right) \otimes_{\mathbb{F}_{q}} \mathbb{F}_{q}\left[\beta_{1}, \ldots, \beta_{h}\right],
$$

where the degrees of $\alpha_{i}$ and of $\beta_{i}$ are 1 and 2, respectively. See [2, Sections V.2, 3, 4] for details.

We will determine a basis of the second cohomology $H_{\mathrm{gr}}^{2}\left(G ; \mathbb{F}_{q}\right)$. We remark that the first one $H_{\mathrm{gr}}^{1}\left(G ; \mathbb{F}_{q}\right) \cong \operatorname{Hom}\left(G, \mathbb{F}_{q}\right) \cong\left(\mathbb{F}_{q}\right)^{h}$ is generated by the Frobenius maps $U_{1}^{p^{i}}$ with $0<i \leq h$. Recall that the cup product is the usual product in the complex $C_{\mathrm{gr}}^{*}\left(G ; \mathbb{F}_{q}\right)$; see [2, Section V.3]. Hence, remembering (24), the cup products $U_{1}^{q_{1}} U_{2}^{q_{2}}$ for $q_{1}<q_{2}<q$ are non-trivial and are linearly independent in $C_{\mathrm{gr}}^{2}\left(G ; \mathbb{F}_{q}\right)$ up to coboundary. For any $z \in \mathbb{F}_{q}$ we set $\mathcal{Z}:=\sum_{n=1}^{p}(z, n z) \in C_{2}^{\mathrm{gr}}\left(G ; \mathbb{F}_{q}\right)$. Then we easily see that $\mathcal{Z}$ is a 2 -cycle, and further compute the pairings

$$
\left\langle U_{1}^{q_{1}} U_{2}^{q_{2}}, \mathcal{Z}\right\rangle=0, \quad\left\langle\chi\left(U_{1}, U_{2}\right)^{q_{3}}, \mathcal{Z}\right\rangle=(-z)^{q_{3} \cdot p} .
$$

Since $z$ is arbitrary, we see the linear independence of these 2-cocycles. Noting that $\operatorname{dim} H_{\mathrm{gr}}^{2}\left(G ; \mathbb{F}_{q}\right)=h(h+1) / 2$, the set in $(23)$ forms a basis of $H_{\mathrm{gr}}^{2}\left(G ; \mathbb{F}_{q}\right)$ as desired.

Next we discuss the third cohomology. Following (24) again, the third cohomology is generated by the products of $H_{\mathrm{gr}}^{1}\left(G ; \mathbb{F}_{q}\right)$ and $H_{\mathrm{gr}}^{2}\left(G ; \mathbb{F}_{q}\right)$. Hence, by the presentation of their cohomologies, the above polynomials give a basis of $H_{\mathrm{gr}}^{3}\left(G ; \mathbb{F}_{q}\right)$ as desired.

Finally, concerning the invariant parts, notice that the boundary $\partial_{*}^{\text {gr }}$ commutes with the action of $\mathbb{Z}$ on $\mathbb{F}_{q}$, by definition. We therefore have a direct decomposition

$$
C_{n}^{\mathrm{gr}}\left(G ; \mathbb{F}_{q}\right) \cong \bigoplus_{i} C_{n}^{\mathrm{gr}}\left(G ; \mathbb{F}_{q}\right)^{\omega^{i}}
$$

as chain groups, where $C_{n}^{\mathrm{gr}}\left(G ; \mathbb{F}_{q}\right)^{\omega^{j}}$ denotes the $\omega^{j}$-eigenvalue space of $C_{n}^{\mathrm{gr}}\left(G ; \mathbb{F}_{q}\right)$. Hence all cocycles of the $\mathbb{Z}$-invariant parts $H_{\mathrm{gr}}^{i}\left(G ; \mathbb{F}_{q}\right)^{\mathbb{Z}}$ are given by ones in a subring of $H_{\mathrm{gr}}^{i}\left(G ; \mathbb{F}_{q}\right)$ as desired.

Returning to our subject, we apply these generators in Lemma 4.4 to the pullback of the chain map $\varphi_{3}$ (see Definition 2.5). Then the quandle 3-cocycles in $I_{q, \omega}^{+}$in (22) explicitly appear as follows:

Lemma 4.5 We have

$$
\begin{aligned}
\varphi_{3}^{*}\left(U_{1}^{q_{1}} U_{2}^{q_{2}} U_{3}^{q_{3}}\right) & =t_{X}\left(1-\omega^{q_{1}}\right)\left(1-\omega^{q_{1}+q_{2}}\right) \cdot U_{1}^{q_{1}} U_{2}^{q_{2}} U_{3}^{q_{3}}, \\
\varphi_{3}^{*}\left(\chi\left(U_{1}, U_{2}\right)^{q_{1}} \cdot U_{3}^{q_{2}}\right) & =t_{X}\left(\omega^{q_{1}}-1\right) \cdot E_{0}\left(p \cdot q_{1}, q_{2}\right), \\
\varphi_{3}^{*}\left(U_{1}^{q_{1}} \cdot \chi\left(U_{2}, U_{3}\right)^{q_{2}}\right) & =t_{X}\left(\omega^{q_{1}}-1\right) \cdot E_{1}\left(q_{1}, p \cdot q_{2}\right) \in C_{Q}^{3}\left(X ; \mathbb{F}_{q}\right) .
\end{aligned}
$$


Proof We will show only the second identity since we can do the others in a similar manner.

Since the 3 -cocycle $\chi\left(U_{1}, U_{2}\right)^{q_{1}} \cdot U_{3}^{q_{2}}$ is $\mathbb{Z}$-invariant by Lemma 4.4 , the sum in the formula (7) becomes the multiplication of $t_{X}$ and it is sufficient to consider the case $i=0$. Hence, according to the formula (7), the left-hand side hand is

$$
\begin{aligned}
t_{X} \cdot\left(\chi\left(U_{1}, U_{2}\right)^{q_{1}}-\chi\left(\omega U_{1}, U_{2}\right)^{q_{1}}-\chi\left(\omega U_{1}, \omega U_{2}\right)^{q_{1}}+\chi\left(\omega^{2} U_{1}, \omega U_{2}\right)^{q_{1}}\right) \cdot U_{3}^{q_{2}} \\
=t_{X}\left(1-\omega^{q_{1}}\right) \cdot\left(\chi\left(U_{1}, U_{2}\right)^{q_{1}}-\chi\left(\omega U_{1}, U_{2}\right)^{q_{1}}\right) \cdot U_{3}^{q_{2}} .
\end{aligned}
$$

Here we use that $\chi\left(\omega U_{1}, \omega U_{2}\right)^{q_{1}}=\omega^{q_{1}} \chi\left(U_{1}, U_{2}\right)^{q_{1}}$ by definition. Since the last term is $t_{X}\left(\omega^{q_{1}}-1\right) \cdot E_{0}\left(q_{1}, q_{2}\right)$ exactly, we obtain the desired identity.

Compared with the method [19] in which the right quandle 3-cocycles were found as solutions of a differential equation over $\mathbb{F}_{q}$, the three identities via the map $\varphi_{3}^{*}$ are simple and miraculous.

Using the identities, we will prove Proposition 2.12 as follows:

Proof of Proposition 2.12 The injectivity of $\Phi_{3}^{*}=\left(\pi_{\rho} \circ \varphi_{3}\right)^{*}$ follows from the fact that $\Phi_{3}^{*}$ gives a $1: 1$ correspondence between a basis of $H_{\mathrm{gr}}^{3}\left(\left(\mathbb{Z}_{p}\right)^{h} ; \mathbb{F}_{q}\right)^{\mathbb{Z}}$ and a basis of a subspace of $H_{Q}^{3}\left(X ; \mathbb{F}_{q}\right)$ because of the previous three identities (compare Theorem 4.3 with Lemma 4.4).

Next assume $H_{Q}^{2}\left(X ; \mathbb{F}_{q}\right)=0$. Then Theorem 4.1 implies that no pair $\left(q_{1}, q_{2}\right)$ satisfies $\omega^{q_{1}+q_{2}}=1$ with $q_{1}<q_{2}<q$. Hence, by examining Theorem 4.3 carefully, we see that $H_{Q}^{3}\left(X ; \mathbb{F}_{q}\right)$ is generated by the image of $\Phi_{3}^{*}$. Therefore $\Phi_{3}^{*}$ is an isomorphism as desired.

To prove Theorem 2.13, we now examine the cokernel $\operatorname{Coker}\left(\Phi_{3}^{*}\right)$. To begin with, we study the chain map

$$
\left(\Phi_{2} \circ \mathcal{P}\right)^{*}: H_{\mathrm{gr}}^{2}\left(\left(\mathbb{Z}_{p}\right)^{h} ; \mathbb{F}_{q}\right)^{\mathbb{Z}} \rightarrow H_{Q}^{3}(X)
$$

from Proposition 2.9. Recall from Lemma 4.4 that this domain is generated by polynomials of the form $U_{1}^{q_{1}} U_{2}^{q_{2}}$. So, recalling the composite $\Phi_{2} \circ \mathcal{P}$ from Proposition 2.9, we easily see

$$
\left(\Phi_{2} \circ \mathcal{P}\right)^{*}\left(U_{1}^{q_{1}} U_{2}^{q_{2}}\right)=t_{X}\left(1-\omega^{q_{1}}\right) U_{1}^{q_{1}} U_{2}^{q_{2}} \in C_{Q_{G}}^{3}\left(X ; \mathbb{F}_{q}\right) .
$$

Hence, the third term in Theorem 4.3 is spanned by the image of this map $\left(\Phi_{2} \circ \mathcal{P}\right)^{*}$. We also discuss the cokernel of $\Phi_{3}^{*} \oplus\left(\Phi_{2} \circ \mathcal{P}\right)^{*}$. By examining Theorem 4.3 carefully, we see that a basis of the cokernel consists of the polynomials $\Gamma$ coming from the 
Mochizuki quadruples $\mathcal{Q}_{q, \omega}$. Let us denote a quadruple $\left(q_{1}, q_{2}, q_{3}, q_{4}\right) \in \mathcal{Q}_{q, \omega}$ by $\mathfrak{q}$ for short. Case by case, we now introduce a map $\theta_{\Gamma}^{\mathfrak{q}}:\left(G_{X}\right)^{3} \rightarrow \mathbb{F}_{q}$ by setting the values of $\theta_{\Gamma}^{\mathfrak{q}}$ at $(x, a \otimes b, y, c \otimes d, z, e \otimes f) \in\left(X \times \operatorname{Coker}\left(\mu_{X}\right)\right)^{3}=\left(G_{X}\right)^{3}$ as follows. In Case $1, \theta_{\Gamma}^{\mathfrak{q}}$ is defined by the formula

$$
\begin{aligned}
(1-\omega)^{-q_{2}}\left(x^{q_{1}} y^{q_{2}+q_{3}}\right. & +x^{q_{1}+q_{3}} y^{q_{2}} \\
& -(1-\omega)^{-q_{2}}\left(\omega^{q_{2}} a^{q_{1}} b^{q_{2}}+a^{q_{2}} b^{q_{1}}-x^{q_{1}+q_{2}}\right) y^{q_{3}} \\
& \left.+(1-\omega)^{-q_{1}}\left(a^{q_{1}} b^{q_{3}}+\omega^{q_{1}} a^{q_{3}} b^{q_{1}}-x^{q_{1}+q_{3}}\right) y^{q_{2}}\right) z^{q_{4}} .
\end{aligned}
$$

In Case 2, the value of $\theta_{\Gamma}^{\mathfrak{q}}$ is given by the formula

$$
\begin{aligned}
(1-\omega)^{-q_{1}-q_{2}}\left(x ^ { q _ { 1 } } \left(y^{q_{2}+q_{3}} z^{q_{4}}\right.\right. & \left.+y^{q_{2}} z^{q_{3}+q_{4}}\right)-\left(x^{q_{1}+q_{2}} y^{q_{4}}+x^{q_{2}} y^{q_{1}+q_{4}}\right) z^{q_{3}} \\
& +(1-\omega)^{-q_{3}}\left(x^{q_{1}+q_{3}}-\omega^{q_{3}} a^{q_{1}} b^{q_{3}}-a^{q_{3}} b^{q_{1}}\right) y^{q_{2}} z^{q_{4}} \\
& \left.-(1-\omega)^{-q_{4}}\left(x^{q_{2}+q_{4}}-\omega^{q_{4}} a^{q_{2}} b^{q_{4}}-a^{q_{4}} b^{q_{2}}\right) y^{q_{1}} z^{q_{3}}\right) .
\end{aligned}
$$

Furthermore, for Case 3 (resp. 4 and 5), the value is defined to be that of Case 1 after changing the indices $(1,2,3,4)$ to $(1,3,4,2)$ (resp. to $(3,1,2,4))$, according to Remark 4.2.

Lemma 4.6 For $\mathfrak{q}=\left(q_{1}, q_{2}, q_{3}, q_{4}\right) \in \mathcal{Q}_{q, \omega}$, the map $\theta_{\Gamma}^{\mathfrak{q}}$ from $\left(G_{X}\right)^{3}$ to $\mathbb{F}_{q}$ is a $\mathbb{Z}$-invariant group 3-cocycle of $G_{X}$.

Moreover, the pullback $\widetilde{\Phi}_{3}^{*}\left(\theta_{\Gamma}^{\mathfrak{q}}\right)$ equals $t_{X} \cdot p_{X}^{*}(\Gamma(\mathfrak{q}))$ in $C_{Q}^{3}\left(\tilde{X} ; \mathbb{F}_{q}\right)$.

Proof Note that a map $\theta:\left(G_{X}\right)^{3} \rightarrow A$ is a $\mathbb{Z}$-invariant group 3-cocycle, by definition, if and only if it satisfies the two equalities

$$
\begin{gathered}
\theta(\mathfrak{b}, \mathfrak{c}, \mathfrak{d})-\theta(\mathfrak{a} \mathfrak{b}, \mathfrak{c}, \mathfrak{d})+\theta(\mathfrak{a}, \mathfrak{b} \mathfrak{c}, \mathfrak{d})-\theta(\mathfrak{a}, \mathfrak{b}, \mathfrak{c} d)+\theta(\mathfrak{a}, \mathfrak{b}, \mathfrak{c})=0 \\
\theta((\omega a, \alpha),(\omega b, \beta),(\omega c, \gamma))=\theta((a, \alpha),(b, \beta),(c, \gamma))
\end{gathered}
$$

for any $\mathfrak{a}=(a, \alpha), \mathfrak{b}=(b, \beta), \mathfrak{c}=(c, \gamma), \mathfrak{d}=(d, \delta) \in G_{X}=X \times \operatorname{Coker}\left(\mu_{X}\right)$. Then, by elementary and direct computations, it can be seen that the maps $\theta_{\Gamma}^{\mathfrak{q}}$ are $\mathbb{Z}$-invariant group 3-cocycles of $G_{X}$. Also, similar to Lemma 4.5, the desired equality $\widetilde{\Phi}_{3}^{*}\left(\theta_{\Gamma}^{\mathfrak{q}}\right)=$ $t_{X} \cdot p_{X}^{*}(\Gamma(\mathfrak{q}))$ is easily obtained by a direct calculation.

Proof of Theorem 2.13. Let $q$ be odd. As is known [22, Lemma 9.15], the induced map $p_{X}^{*}: H_{Q}^{3}\left(X ; \mathbb{F}_{q}\right) \rightarrow H_{Q}^{3}\left(\tilde{X} ; \mathbb{F}_{q}\right)$ is surjective. Hence, Lemma 4.6 ensures the existence of a section

$$
\mathfrak{s}: H_{Q}^{3}\left(\tilde{X} ; \mathbb{F}_{q}\right) \rightarrow H_{Q}^{3}\left(X ; \mathbb{F}_{q}\right)
$$


such that $\mathfrak{s}\left(\widetilde{\Phi}_{3}^{*}\left(\theta_{\Gamma}^{\mathfrak{q}}\right)\right)=\Gamma(\mathfrak{q})$ for any $\mathfrak{q} \in \mathcal{Q}_{q, \omega}$. Thus the sum

$$
\left(\left(\Phi_{2} \circ \mathcal{P}\right)^{*} \oplus \Phi_{3}^{*}\right) \oplus\left(\mathfrak{s} \circ \operatorname{res}\left(\widetilde{\Phi}_{3}^{*}\right)\right)
$$

explained in (8) is an isomorphism to $H_{Q}^{3}\left(X ; \mathbb{F}_{q}\right)$.

We will show that the above group 3-cocycles $\theta_{\Gamma}^{\mathfrak{q}}$, except the ones from Case 2, are presented by Massey products. To see this, we consider a group homomorphism

$$
f^{q_{i}}: G_{X} \rightarrow \mathbb{F}_{q}, \quad(x, \alpha) \mapsto x^{q_{i}},
$$

which is a group 1-cocycle of $G_{X}$. For group 1-cocycles $f, g$ and $h$, we denote by $f \wedge g$ the cup product; also, if $f \wedge g=g \wedge h=0 \in H_{\mathrm{gr}}^{2}\left(G_{X} ; \mathbb{F}_{q}\right)$, we denote by $\langle f, g, h\rangle$ the triple Massey product in $H_{\mathrm{gr}}^{2}\left(G_{X} ; \mathbb{F}_{q}\right)$ as usual (see Kraines [15] for the definition).

Proposition 4.7 Let $e \neq 2$. Let $\left(q_{1}, q_{2}, q_{3}, q_{4}\right) \in \mathcal{Q}_{q, \omega}$ satisfy Case $e$ in Section 4.1. The group 3-cocycle $\theta_{\Gamma}^{\mathfrak{q}}$ described above is of the following form in the cohomology group $H_{\mathrm{gr}}^{3}\left(G_{X} ; \mathbb{F}_{q}\right)$.

$$
H_{\mathrm{gr}}^{3}\left(G_{X} ; \mathbb{F}_{q}\right) \ni \theta_{\Gamma}^{\mathfrak{q}}= \begin{cases}\left(1-\omega^{q_{2}}\right)^{-1}\left\langle f^{q_{3}}, f^{q_{1}}, f^{q_{2}}\right\rangle \wedge f^{q_{4}} & \text { for } e=1, \\ \left(1-\omega^{q_{3}}\right)^{-1}\left\langle f^{q_{4}}, f^{q_{1}}, f^{q_{3}}\right\rangle \wedge f^{q_{2}} & \text { for } e=3, \\ \left(1-\omega^{q_{3}}\right)^{-1}\left\langle f^{q_{1}}, f^{q_{2}}, f^{q_{3}}\right\rangle \wedge f^{q_{4}} & \text { for } e=4 \text { or } 5 .\end{cases}
$$

Proof We use notation $(x, a \otimes b, y, c \otimes d, z, e \otimes f) \in\left(X \times \operatorname{Coker}\left(\mu_{X}\right)\right)^{3}$ as above. For Case 1 , we now calculate the Massey product $\left\langle f^{q_{3}}, f^{q_{1}}, f^{q_{2}}\right\rangle$. We easily check the two equalities

$$
\begin{aligned}
& x^{q_{3}} y^{q_{1}}=(1-\omega)^{-q_{1}} \delta_{1}\left(a^{q_{1}} b^{q_{3}}+\omega^{q_{1}} a^{q_{3}} b^{q_{1}}-x^{q_{1}+q_{3}}\right), \\
& x^{q_{1}} y^{q_{2}}=(1-\omega)^{-q_{2}} \delta_{1}\left(\omega^{q_{2}} a^{q_{1}} b^{q_{2}}+a^{q_{2}} b^{q_{1}}-x^{q_{1}+q_{2}}\right) .
\end{aligned}
$$

Hence, from the definition of Massey products, $\left\langle f^{q_{3}}, f^{q_{1}}, f^{q_{2}}\right\rangle$ is represented by

$$
\begin{aligned}
(1-\omega)^{-q_{1}}\left(a^{q_{1}} b^{q_{3}}+\omega^{q_{1}} a^{q_{3}} b^{q_{1}}-x^{q_{1}+q_{3}}\right) y^{q_{2}} & \\
& +(1-\omega)^{-q_{2}} x^{q_{3}}\left(\omega^{q_{2}} c^{q_{1}} d^{q_{2}}+c^{q_{2}} d^{q_{1}}-y^{q_{1}+q_{2}}\right) .
\end{aligned}
$$

Furthermore, we define a group 2-cocycle $\mathcal{F}$ by the formula

$$
(1-\omega)^{-q_{2}}\left(\left\langle f^{q_{3}}, f^{q_{1}}, f^{q_{2}}\right\rangle+(1-\omega)^{-q_{2}} \delta_{1}\left(\omega^{q_{2}} x^{q_{3}} a^{q_{1}} b^{q_{2}}+x^{q_{3}} a^{q_{2}} b^{q_{1}}-x^{q_{1}+q_{2}+q_{3}}\right)\right) .
$$

A direct calculation then shows the equality $\mathcal{F} \cdot z^{q_{4}}=\theta_{\Gamma}^{\mathfrak{q}}$ by definitions, immediately leading to the desired $(1-\omega)^{-q_{1}}\left\langle f^{q_{3}}, f^{q_{1}}, f^{q_{2}}\right\rangle \wedge f^{q_{4}}=\theta_{\Gamma}^{\mathfrak{q}} \in H_{\mathrm{gr}}^{3}\left(G_{X} ; \mathbb{F}_{q}\right)$.

Similarly, the same calculation holds for Cases 3, 4, 5 according to Remark 4.2.

An algebraic interpretation of the cocycle $\theta_{\Gamma}^{\mathfrak{q}}$ in Case 2 remains to be found. 


\section{Some calculations of shadow cocycle invariants}

As an application of Theorem 2.15, we will compute some $\mathbb{Z}$-equivariant parts of the Dijkgraaf-Witten invariants, which are equivalent to a shadow cocycle invariant. In this section, we restrict ourselves to the Alexander quandles on $\mathbb{F}_{q}$ with $\omega \in \mathbb{F}_{q}$. Recall from Lemma 4.6 that the quandle 3-cocycles $\Gamma\left(q_{1}, q_{2}, q_{3}, q_{4}\right)$ found by Mochizuki (see Section 4.1 for the definition) are derived not from group cohomologies of abelian groups, but from that of the non-abelian group $G_{X}$. So we focus on the 3-cocycles, and fix some notation: for short, let $\mathfrak{q}$ denote a Mochizuki quadruple $\left(q_{1}, q_{2}, q_{3}, q_{4}\right)$ in $\mathcal{Q}_{q, \omega}$, and let $\Gamma(\mathfrak{q})_{e}$ denote the 3 -cocycle $\Gamma\left(q_{1}, q_{2}, q_{3}, q_{4}\right)$, if $\mathfrak{q}$ satisfies Case $e$ in Section $4.1(e \leq 5)$.

We remark that the set of $X$-colorings has been well-studied. In fact, if $D$ is a diagram of a knot $K$, then there is a bijection

$$
\operatorname{Col}_{X}(D) \leftrightarrow X \oplus \bigoplus_{i=1} \mathbb{F}_{q}[T] /\left(T-\omega, \Delta_{i}(T) / \Delta_{i+1}(T)\right),
$$

where $\Delta_{i}(T)$ is the $i^{\text {th }}$ Alexander polynomial of $K$ (see Inoue [10]). Therefore, we shall study weights in the cocycle invariants.

\subsection{Cocycle invariants of torus knots constructed from $\Gamma\left(q_{1}, q_{2}, q_{3}, q_{4}\right)$}

This subsection deals with the torus knots $T(m, n)$. We here remark that $m$ and $n$ are relatively prime and there is an isotopy $T(m, n) \simeq T(n, m)$, so we may assume $n$ is relatively prime to $p$ without loss of generality. We determine all of the values of the invariants for $T(m, n)$ as follows: ${ }^{4}$

Theorem 5.1 Let $q$ be relatively prime to $n$. Take the torus knot $T(m, n)$. Let $\mathfrak{q} \in \mathcal{Q}_{q, \omega}$ be a Mochizuki quadruple, and $\Gamma(\mathfrak{q})_{e}$ be the associated quandle 3-cocycle. Then the quandle cocycle invariant $I_{\Gamma(\mathfrak{q})_{e}}(T(m, n))$ is expressed by one of the following formulas:

(i) If $e=1, \omega^{m n}=1, \omega^{m} \neq 1$ and $\omega^{n} \neq 1$, then $I_{\Gamma(\mathfrak{q})_{1}}(T(m, n))$ equals

$$
q^{2} \sum_{a \in \mathbb{F}_{q}} 1_{\mathbb{Z}}\left\{-2 m n \frac{(\zeta-\omega)^{q_{2}+q_{3}} \omega^{q_{4}}}{(1-\zeta)^{q_{2}+q_{3}}} \cdot a^{q_{1}+q_{2}+q_{3}+q_{4}}\right\} \in \mathbb{Z}\left[\mathbb{F}_{q}\right],
$$

where $\zeta$ is the $n^{\text {th }}$ primitive root of unity satisfying $\omega^{m}=\zeta^{m}$. Furthermore, if $e=3$ (resp. 4 or 5 ), then the value of $I_{\Gamma(\mathfrak{q})_{e}}$ is obtained from the above value

${ }^{4}$ We here refer to known results of Asami and Kuga [1, Section 5.2]. They partially calculated some values of $I_{\Gamma(\mathfrak{q})_{e}}(T(m, n))$ in the case $\mathbb{F}_{q}=\mathbb{F}_{5^{2}}$ and $n=3$, with the help of a computer. 
$I_{\Gamma(\mathfrak{q})_{1}}$ after changing the indices $(1,2,3,4)$ to $(1,3,4,2)$ (resp. to $\left.(3,1,2,4)\right)$ such as Remark 4.2.

(ii) Let $p=2$ or 3 , and let $e=1$. If $\omega^{n}=1$ and if $m$ is divisible by $p$, then

$$
I_{\Gamma(\mathfrak{q})_{1}}(T(m, n))=q^{2} \sum_{a \in \mathbb{F}_{q}} 1_{\mathbb{Z}}\left\{\frac{m n}{p}(1-\omega)^{q_{3}+q_{4}} a^{q_{1}+q_{2}+q_{3}+q_{4}}\right\} \in \mathbb{Z}\left[\mathbb{F}_{q}\right] .
$$

Furthermore, if $e=3$ (resp. 4 or 5 ), then the value $I_{\Gamma(\mathfrak{q})}$ is obtained from the value $I_{\Gamma(\mathfrak{q})_{1}}$ after changing the indices $(1,2,3,4)$ to $(1,3,4,2)$ (resp. to $(3,1,2,4))$, similarly.

(iii) Let $e=2$. If $p=2, \omega^{n}=1$ and if $m$ is divisible by 2 , then $I_{\Gamma(\mathfrak{q})_{2}}(T(m, n))$ is equal to $q \sum_{a, \delta \in \mathbb{F}_{q}} 1_{\mathbb{Z}}\left\{m n \mathcal{E}_{2}(a, \delta) / 2\right\} \in \mathbb{Z}\left[\mathbb{F}_{q}\right]$. Here $\mathcal{E}_{2}(a, \delta) \in \mathbb{F}_{q}$ is temporarily defined by

$$
\begin{aligned}
a^{q_{2}+q_{3}}\left(\left(1+\omega^{q_{1}}\right) a^{q_{1}} \delta^{q_{4}}+(1\right. & \left.\left.+\omega^{q_{4}}\right) a^{q_{4}} \delta^{q_{1}}\right) \\
& +a^{q_{1}+q_{4}}\left(\left(1+\omega^{q_{2}}\right) a^{q_{2}} \delta^{q_{3}}+\left(1+\omega^{q_{3}}\right) a^{q_{3}} \delta^{q_{2}}\right) .
\end{aligned}
$$

(iv) Otherwise, the invariant is trivial. Namely, $I_{\Gamma(\mathfrak{q})_{e}}(T(m, n)) \in \mathbb{Z}$.

This is proved in Section 5.3. Note that for $e=2$, the invariant is non-trivial in only the case (iii).

As an application, we consider the $t$-fold cyclic cover of $S^{3}$ branched over $T(m, n)$, which is the Brieskorn manifold $\Sigma(m, n, t)$; see Milnor [18]. Hence, according to Remark 2.16, we obtain the $\mathbb{Z}$-equivariant part of the Dijkgraaf-Witten invariant of $\Sigma(m, n, t)$.

Corollary 5.2 Let $m, n$ be coprime integers. Assume that $X$ is of type $t$. Let a Mochizuki quadruple $\left(q_{1}, q_{2}, q_{3}, q_{4}\right) \in \mathcal{Q}_{q, \omega}$ satisfy Case 1 , and $\theta_{\Gamma} \in H_{\mathrm{gr}}^{3}\left(G_{X} ; \mathbb{F}_{q}\right)$ be the group 3-cocycle in Lemma 4.6. Let $p>2$ be coprime to $n$ and to $t$. If $\omega^{m n}=1$, $\omega^{n} \neq 1$ and $\omega^{m} \neq 1$, then

$$
\operatorname{DW}_{\theta_{\Gamma}}^{\mathbb{Z}}(\Sigma(m, n, t))=\sum_{a \in \mathbb{F}_{q}} 1_{\mathbb{Z}}\left\{-2 t m n \frac{(\zeta-\omega)^{q_{2}+q_{3}} \omega^{q_{4}}}{(1-\zeta)^{q_{2}+q_{3}}} a^{q_{1}+q_{2}+q_{3}+q_{4}}\right\} \in \mathbb{Z}\left[\mathbb{F}_{q}\right]
$$

Here recall from Proposition 4.7 that the cocycle $\theta_{\Gamma}$ forms a Massey product; hence we clarify partially the Massey product structure of some Brieskorn manifolds. Here we emphasize that there are a few methods for computing Massey products with $\mathbb{Z} / p$-coefficients, in comparison with those with $\mathbb{Q}$-coefficients viewed from rational homotopy theory. 
Finally, we comment on the interesting result in Theorem 5.1(ii). In general, for finite nilpotent groups $G$, the Massey products in $H_{\mathrm{gr}}^{3}\left(G ; \mathbb{F}_{q}\right)$ with $p=2,3$ often display exceptional phenomena. For example, when $q=p^{2}$, the group $G_{X}$ is isomorphic to the group $P(3)$ in Leary [16]. See [16, Theorems 6 and 7] for an exceptional phenomenon in the cohomology ring $H_{\mathrm{gr}}^{*}\left(G_{X} ; \mathbb{F}_{p}\right)$ with $p=2,3$.

\subsection{Further examples in the case $\omega=-1$}

We change our focus to other knots. However, it is not so easy to calculate the cocycle invariant $I_{\Gamma(\mathfrak{q})_{e}}(K)$ of knots, although it is elementary.

We now consider the simplest case $\omega=-1$; hence the Alexander quandle $X$ on $\mathbb{F}_{q}$ is of type 2 . Note that, for any Mochizuki quadruple $\mathfrak{q}=\left(q_{1}, q_{2}, q_{3}, q_{4}\right)$, the associated 3-cocycle forms $U_{1}^{q_{1}} U_{2}^{q_{2}+q_{3}} U_{3}^{q_{4}}$ by definition. Thus it is not relatively hard to compute the cocycle invariant. However, in computer experiments, for many knots whose colorings satisfy $\left|\operatorname{Col}_{X}(D)\right|=q^{2}$, the invariants are frequently of the form $q^{2} \sum_{a \in \mathbb{F}_{q}} a^{q_{1}+q_{2}+q_{3}+q_{4}}$ up to constant factors. In order to avoid the cases $\left|\operatorname{Col}_{X}(D)\right|=q$ and $\left|\operatorname{Col}_{X}(D)\right|=q^{2}$, recall the bijection (27). Accordingly, we shall deal with some knots having non-trivial second Alexander polynomials as follows:

Example 5.3 Let $\omega=-1$. The knots $K$ in Table 1 are those whose crossing numbers are less than 11 , satisfying $\left|\operatorname{Col}_{X}(D)\right|=q^{3}$ with $p>3$, and whose second Alexander polynomials are non-trivial. We only list computations of the invariants without the proofs, although the computations seem interesting. Here note that, according to Theorem 2.15 and Proposition 4.7, the cocycle invariant stems from triple Massey products of double branched covering spaces. We refer to the tables in Kawauchi [14, Exercise 5.5.5, Appendix F.2] for some information of double coverings of $S^{3}$ branched over such knots $K$.

\begin{tabular}{ccc}
$K$ & $p$ & $I_{\Gamma(\mathfrak{q})_{1}}(K)$ \\
\hline $9_{40}$ & 5 & $\mathcal{G}(\mathfrak{q} ; 1,5)$ \\
$9_{41}$ & 7 & $\mathcal{G}(\mathfrak{q} ; 3,4)$ \\
$9_{49}$ & 5 & $\mathcal{G}(\mathfrak{q} ; 3,4)$ \\
$10_{103}$ & 5 & $\mathcal{G}(\mathfrak{q} ; 2,1)$ \\
$10_{123}$ & 11 & $q^{4}$ \\
$10_{155}$ & 5 & $\mathcal{G}_{155}(\mathfrak{q})$ \\
$10_{157}$ & 7 & $\mathcal{G}(\mathfrak{q} ; 1,5)$
\end{tabular}

Table 1: The values of $I_{\Gamma(\mathfrak{q})_{1}}(K)$ 
Here $\mathfrak{q} \in \mathcal{Q}_{q, \omega}$ is arbitrary and, for $n, m \in \mathbb{Z}$, the symbols $\mathcal{G}(\mathfrak{q} ; n, m)$ and $\mathcal{G}_{155}(\mathfrak{q})$ are polynomials expressed by

$$
\begin{aligned}
\mathcal{G}(\mathfrak{q} ; n, m):=q^{2} \sum_{a, b \in \mathbb{F}_{q}} 1_{\mathbb{Z}}\left\{n \left(a^{q_{1}+q_{2}} b^{q_{3}}+q_{4}\right.\right. & +a^{q_{3}+q_{4}} b^{q_{1}+q_{2}} \\
+ & \left.a^{q_{1}+q_{3}} b^{q_{2}+q_{4}}+a^{q_{2}+q_{4}} b^{q_{1}+q_{3}}\right) \\
+ & \left.m\left(a^{q_{1}+q_{4}} b^{q_{2}+q_{3}}+a^{q_{2}+q_{3}} b^{q_{1}+q_{4}}\right)\right\} \in \mathbb{Z}\left[\mathbb{F}_{q}\right], \\
\mathcal{G}_{155}(\mathfrak{q}):=q^{2} \sum_{a, b \in \mathbb{F}_{q}} 1_{\mathbb{Z}}\left\{4 \left(a^{q_{1}+q_{2}+q_{3}+q_{4}}\right.\right. & \left.+a^{q_{1}} b^{q_{2}+q_{3}+q_{4}}\right) \\
& +\left(a^{q_{1}+q_{2}+q_{3}} b^{q_{4}}+a^{q_{1}+q_{2}} b^{q_{3}+q_{4}}\right) \\
& +2\left(a^{q_{1}+q_{2}+q_{4}} b^{q_{2}}+a^{q_{1}+q_{2}+q_{4}} b^{q_{3}}\right. \\
& \left.\left.+a^{q_{1}+q_{3}} b^{q_{2}+q_{4}}+a^{q_{2}+q_{4}} b^{q_{1}+q_{3}}\right)\right\} \in \mathbb{Z}\left[\mathbb{F}_{q}\right] .
\end{aligned}
$$

\subsection{Proof of Theorem 5.1}

For the proof, we first recall a slight reduction [11, Theorem 4.3] of the cocycle invariant, which implies that the shadow cocycle invariant does not depend on the complementary containing the infinity point. That is, we may consider only shadow colorings of the forms $\mathcal{S}=(\mathcal{C} ; 0)$. More precisely,

$$
I_{\psi}(L)=q \cdot \sum_{\mathcal{C} \in \operatorname{Col}_{X}(D)} 1_{\mathbb{Z}}\{\langle\psi,[(\mathcal{C} ; 0)]\rangle\} \in \mathbb{Z}[A] .
$$

We establish terminologies on the torus knot $T(m, n)$. Regard $T(m, n)$ as the closure of a braid $\Delta^{m}$, where $\Delta:=\sigma_{n-1} \cdots \sigma_{1} \in B_{n}$. Let $\alpha_{1}, \ldots, \alpha_{n}$ be the top arcs of $\Delta^{m}$. For $1 \leq i \leq m$, we let $x_{i, 1}, \ldots, x_{i, n-1}$ be the crossings in the $i^{\text {th }} \Delta$; see Figure 4 .

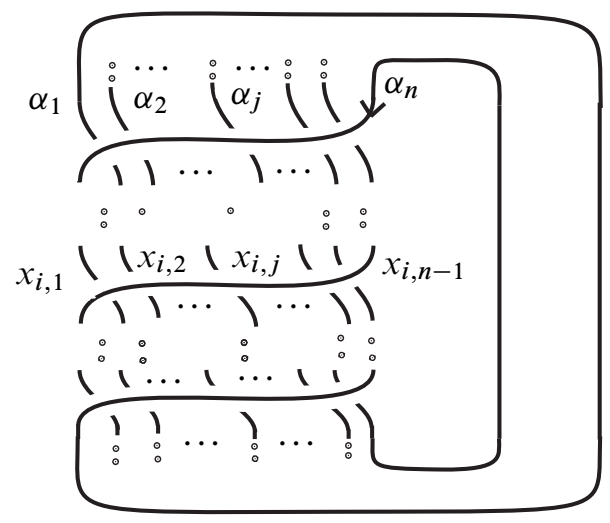

Figure 4: The arcs $\alpha_{j}$ and crossing points $x_{i, j}$ on the diagram of the torus knot 
Proof of Theorem 5.1 Although Asami and Kuga [1] formulated some $X$-colorings of $T(m, n)$, we will give another formulation appropriate to the 3-cocycle $\Gamma(\mathfrak{q})_{e}$. If given an $X$-coloring $\mathcal{C}$ of $T(m, n)$, we define $a_{j}:=\mathcal{C}\left(\alpha_{j}\right)$, and consider a vector $\boldsymbol{a}=\left(a_{1}, \ldots, a_{n}\right) \in\left(\mathbb{F}_{q}\right)^{n}$; notice that it satisfies the equation $\boldsymbol{a}=\boldsymbol{a} P^{m}$, where $P$ is given by a companion matrix

$$
P:=\left(\begin{array}{cccccc}
0 & \omega & 0 & \cdots & 0 & 0 \\
0 & 0 & \omega & \cdots & 0 & 0 \\
0 & 0 & 0 & \ddots & 0 & 0 \\
\vdots & \vdots & \vdots & \ddots & \ddots & \vdots \\
0 & 0 & 0 & \cdots & 0 & \omega \\
1 & 1-\omega & 1-\omega & \cdots & 1-\omega & 1-\omega
\end{array}\right) \in \operatorname{Mat}\left(n \times n ; \mathbb{F}_{q}\right) .
$$

Note that the characteristic polynomial of $P$ is $(\lambda-1)\left(\lambda^{n}-\omega^{n}\right) /(\lambda-\omega)$, and that the roots are $\lambda=\zeta^{k} \omega$ and 1 , where $1 \leq k<n$ and by $\zeta$ we mean an $n^{\text {th }}$ primitive root of unity in the algebraic closure $\overline{\mathbb{F}}_{p}$. Therefore, the proof comes down to the following two cases:

Case I $\omega^{n} \neq 1$ To be precise, the roots are mutually distinct.

Case II $\omega^{n}=1$ Then $\lambda=1$ is the unique double root of the characteristic polynomial.

We will calculate the weights coming from such $X$-colorings case by case. While statement (i) will be derived from Case I, (ii) and (iii) will come from Case II.

Case I Let $\omega^{n} \neq 1$. We will study the solutions of $\boldsymbol{a}=\boldsymbol{a} P^{m}$. We easily see that if $\left(\zeta^{-k} \omega\right)^{m}=1$ for some $k$, then the solution is of the form

$$
a_{j+1}=a\left(\left(1-\zeta^{k j}\right) /(1-\zeta)\right)+a\left(\zeta^{k j} /(1-\omega)\right)+\delta
$$

for some $a, \delta \in \overline{\mathbb{F}}_{p}$; conversely, if the equation $\boldsymbol{a}=\boldsymbol{a} P^{m}$ has a non-trivial solution, then there is a unique $k$ satisfying $\left(\zeta^{-k} \omega\right)^{m}=1$ and $0<k<n$. It is further verified that such a solution gives rise to an $X$-coloring $\mathcal{C}$ if and only if $a, \delta, \zeta$ are contained in $\mathbb{F}_{q}$. To summarize, we may assume that $a, \delta, \zeta \in \mathbb{F}_{q}$ and $\left(\zeta^{-1} \omega\right)^{m}=1$ with $\zeta \neq \omega$. Indeed this assumption justifies a shadow coloring $\mathcal{S}$ of the form $(\mathcal{C} ; 0)$.

Remark 5.4 We give a remark on this assumption. Notice that for $s \in \mathbb{Z}$, two equalities $\omega^{m}=\zeta^{m}$ and $\omega^{s}=1$ imply $\zeta^{m s}=1$, hence $\zeta^{s}=1$ since $m$ and $n$ are coprime. In particular, considering special cases of $s=q_{1}+q_{3}$ and $s=q_{2}+q_{4}$, we have $\zeta^{q_{1}+q_{3}}=\zeta^{q_{2}+q_{4}}=1$. Similarly we notice that if $\omega^{q_{1}+q_{2}}=1$, then $\zeta^{q_{1}+q_{2}}=1$. 
We will present the weights of $[\mathcal{S}]=[(\mathcal{C} ; 0)]$, where $\mathcal{C}$ is the $X$-coloring as the solution mentioned above. We then can easily check the color of every regions in the linkdiagram. After a tedious calculation, the weight of $x_{i, j}$ turns out to be

$$
\left(a \zeta^{-i} \omega^{i} \frac{1-\zeta^{j-1}}{1-\zeta}+\left(1-\omega^{j-1}\right) \delta, a \zeta^{-i} \omega^{i}\left(\frac{1-\zeta^{j-1}}{1-\zeta}+\frac{\zeta^{j-1}}{1-\omega}\right)+\delta, \frac{a \zeta^{-i-1} \omega^{i+1}}{1-\omega}+\delta\right)
$$

in $C_{3}^{Q}(X)$. We next compute the pairing $\left\langle\Gamma(\mathfrak{q})_{e},[\mathcal{S}]\right\rangle \in \mathbb{F}_{q}$. To begin with the case $e=1$, recalling that $\Gamma(\mathfrak{q})_{e}=U_{1}^{q_{1}} U_{2}^{q_{2}+q_{3}} U_{3}^{q_{4}}=\left(x_{1}-x_{2}\right)^{q_{1}}\left(x_{2}-x_{3}\right)^{q_{2}+q_{3}} x_{3}^{q_{4}}$ by definition, we describe the pairing as

$$
\sum_{\substack{i \leq m \\ j \leq n-1}}\left(\frac{a \zeta^{-i} \omega^{i} \zeta^{j-1}}{1-\omega}-\omega^{j-1} \delta\right)^{q_{1}}\left(\frac{a \zeta^{-i-1} \omega^{i}(\omega-\zeta)\left(\zeta^{j}-1\right)}{(1-\omega)(1-\zeta)}\right)^{q_{2}+q_{3}}\left(\frac{a \zeta^{-i-1} \omega^{i+1}}{1-\omega}+\delta\right)^{q_{4}}
$$

in $\mathbb{F}_{q}$. We note that $\sum_{i=1}^{m}\left(\zeta^{-1} \omega\right)^{s i}=0$ unless $\zeta^{-s} \omega^{s}=1$. Therefore, several terms in this formula vanish by Remark 5.4 above. In the sequel, it is easily seen that the non-vanishing term in $\left\langle\Gamma(\mathfrak{q})_{1},[\mathcal{S}]\right\rangle$ forms

$$
\frac{a^{q_{1}+q_{2}+q_{3}+q_{4}}(\zeta-\omega)^{q_{2}+q_{3}} \omega^{q_{4}}}{(1-\omega)^{q_{1}+q_{2}+q_{3}+q_{4}}(1-\zeta)^{q_{2}+q_{3}}} \sum_{\substack{i \leq m \\ j \leq n-1}}\left(\zeta^{-1} \omega\right)^{i\left(q_{1}+q_{2}+q_{3}+q_{4}\right)} \zeta^{j q_{1}}\left(1-\zeta^{j}\right)^{q_{2}+q_{3}},
$$

in $\mathbb{F}_{q}$. Here, by Remark 5.4 again, we notice two equalities

$$
\left(\zeta^{-1} \omega\right)^{q_{1}+q_{2}+q_{3}+q_{4}}=1 \quad \text { and } \quad \zeta^{j q_{1}}\left(1-\zeta^{j}\right)^{q_{2}+q_{3}}=\zeta^{j q_{1}}+\zeta^{j q_{2}}-2 .
$$

Therefore, noting that $\sum_{j=1}^{n-1} \zeta^{j q_{1}}=\sum_{j=1}^{n-1} \zeta^{j q_{2}}=-1$, the sum in the formula (31) equals $-2 n m$. By (30), we hence obtain the required formula (28).

Similarly, by Remark 4.2, the same calculations hold for the cases $3 \leq e \leq 5$.

Next we deal with $e=2$. For the shadow coloring $\mathcal{S}=(\mathcal{C} ; 0)$, we claim $\left\langle\Gamma(\mathfrak{q})_{2},[\mathcal{S}]\right\rangle=0$. To see this, by a similar calculation to $(31)$, we can reduce the paring $\left\langle\Gamma(\mathfrak{q})_{2},[\mathcal{S}]\right\rangle$ to $\left\langle\Gamma(\mathfrak{q})_{2},[\mathcal{S}]\right\rangle=-2 n m a^{q_{1}+q_{2}+q_{3}+q_{4}} \cdot \mathcal{A}_{\mathfrak{q}}$, where $\mathcal{A}_{\mathfrak{q}}$ is temporarily defined by

$$
\frac{(\zeta-\omega)^{q_{2}+q_{3}} \omega^{q_{4}}}{(1-\zeta)^{q_{2}+q_{3}}}-\frac{(\zeta-\omega)^{q_{1}+q_{4}} \omega^{q_{3}}}{(1-\zeta)^{q_{1}+q_{4}}}+\frac{1-\omega^{q_{1}+q_{2}}}{1-\omega^{q_{2}}}\left(\frac{(\zeta-\omega)^{q_{2}} \omega^{q_{3}+q_{4}}}{(1-\zeta)^{q_{2}}}-\frac{(\zeta-\omega)^{q_{4}} \omega^{q_{3}}}{(1-\zeta)^{q_{4}}}\right) .
$$

We assert that the last term in this formula $\mathcal{A}_{\mathfrak{q}}$ is zero. Indeed, noting that $(1-\zeta)^{-q_{4}}=$ $\zeta^{q_{2}}(1-\zeta)^{-q_{4}} \zeta^{-q_{2}}=\zeta^{q_{2}}(\zeta-1)^{-q_{2}}$ by Remark 5.4, we easily have

$$
\frac{(\zeta-\omega)^{q_{2}} \omega^{q_{3}+q_{4}}}{(1-\zeta)^{q_{2}}}-\frac{(\zeta-\omega)^{q_{4}} \omega^{q_{3}}}{(1-\zeta)^{q_{4}}}=\frac{(\zeta-\omega)^{q_{2}} \omega^{q_{3}+q_{4}}+(\zeta-\omega)^{q_{4}} \zeta^{q_{2}} \omega^{q_{3}}}{(1-\zeta)^{q_{2}}}=0
$$


Similarly we easily see an equality $(1-\zeta)^{-q_{1}-q_{4}}=\zeta^{q_{2}+q_{3}}(1-\zeta)^{-q_{2}-q_{3}}$. Therefore the first and second terms in $\mathcal{A}_{\mathfrak{q}}$ are canceled. Hence $\mathcal{A}_{\mathfrak{q}}=0$ as claimed. In conclusion, the cocycle invariants using $\Gamma(\mathfrak{q})_{2}$ are trivial as desired.

Case II We next consider another case of $\omega^{n}=1$. Notice that the matrix $P-E_{n}$ is of rank $n-1$. Hence, if the above equation $\boldsymbol{a}=\boldsymbol{a} P^{m}$ has a non-trivial solution, then $m$ must be divisible by $p$ (consider the Jordan block of $P$ ). For such an $m$, we can verify that the solution is of the form $a_{j}=a \omega-a \omega^{j}+\delta$ for some $a, \delta \in \mathbb{F}_{q}$, which provides an $X$-coloring $\mathcal{C}$. Consider a shadow coloring of the form $\mathcal{S}=(\mathcal{C} ; 0)$. The weight of the crossing $x_{i, j}$ is then given by

$$
\left(a(1-j)(1-\omega) \omega^{j-1}+(a i \omega-a i+a+\delta)\left(1-\omega^{j-1}\right), a\left(1-\omega^{j}+i \omega-i\right)+\delta, a i(\omega-1)+\delta\right) .
$$

Let us calculate the pairings $\left\langle\Gamma(\mathfrak{q})_{e},[\mathcal{S}]\right\rangle$. First, when $e=1$, the pairing $\left\langle\Gamma(\mathfrak{q})_{1},[\mathcal{S}]\right\rangle$ equals

$$
\sum_{\substack{i \leq m \\ j \leq n-1}}\left((a j(\omega-1)-a i(\omega-1)-\delta) \omega^{j-1}\right)^{q_{1}}\left(a-a \omega^{j}\right)^{q_{2}+q_{3}}(a i(\omega-1)+\delta)^{q_{4}} .
$$

We consider the sum on $i$ and note $\sum_{i \leq m} i^{q_{1}+q_{4}}=\sum_{i \leq m} i^{2}=m(m+1)(2 m+1) / 6$. Hence, since $m$ is divisible by $p$, the pairing vanishes unless $p=2,3$.

Similarly, we can see that in other cases of $e$, the pairings are zero unless $p=2,3$. We therefore may devote ourselves to the cases $p=2,3$ hereafter.

First, assume $p=3$ and $e=1$. Note that the non-vanishing term in (32) is only the coefficients of $\sum i^{q_{1}+q_{4}}$, and that $\sum_{i \leq m} i^{q_{1}+q_{4}}=-m / 3$. Then the pairing (32) is reduced to

$$
\begin{aligned}
a^{q_{1}+q_{2}+q_{3}+q_{4}}(1-\omega)^{q_{1}+q_{4}} \sum_{1 \leq j \leq n-1} \omega^{q_{1}(j-1)}\left(1-\omega^{j}\right)^{q_{2}+q_{3}} \sum_{1 \leq i \leq m} i^{q_{1}+q_{4}} \\
=\frac{m n}{3} a^{q_{1}+q_{2}+q_{3}+q_{4}}(1-\omega)^{q_{3}+q_{4}}
\end{aligned}
$$

where $\sum \omega^{q_{1}(j-1)}\left(1-\omega^{j}\right)^{q_{2}+q_{3}}=2 n \omega^{-q_{1}}$ in this equality follows from $\omega^{n}=1$. Hence, by running over all shadow colorings, we obtain the required formula (29). Similarly, when $p=2$ and $e=1$, a calculation using Lemma 5.5(i) below can show the formula (29).

The same calculation holds for the cases $3 \leq e \leq 5$ and $p=2,3$. Actually, it is done by changing the quadruple $\left(q_{1}, q_{2}, q_{3}, q_{4}\right)$ in the previous calculation in Case 1 , as is routine for these cases. 
At last, it is enough for the proof to work out the remaining case $e=2$ and $p=2,3$. By Lemma 5.5(ii) below and the definition of $\Gamma(\mathfrak{q})_{2}$, the pairing is reduced to

$$
\left\langle\Gamma(\mathfrak{q})_{2},[\mathcal{S}]\right\rangle=\left\langle U_{1}^{q_{1}} U_{2}^{q_{2}+q_{3}} U_{3}^{q_{4}},[\mathcal{S}]\right\rangle-\left\langle U_{1}^{q_{2}} U_{2}^{q_{1}+q_{4}} U_{3}^{q_{3}},[\mathcal{S}]\right\rangle .
$$

We claim that if $p=3,\left\langle\Gamma(\mathfrak{q})_{2},[\mathcal{S}]\right\rangle=0$. The first term is reduced to

$$
2 m n a^{q_{1}+q_{2}+q_{3}+q_{4}}(1-\omega)^{q_{3}+q_{4} / 3},
$$

by a calculation similar to (29). The second term is obtained by changing the indices $(1,2,3,4)$ in the first term to $(2,1,4,3)$. Hence the pairing $\left\langle\Gamma(\mathfrak{q})_{2},[\mathcal{S}]\right\rangle$ vanishes.

To complete the proofs, we let $p=2$. The explicit formula of the first term in (33) follows from Lemma 5.5(iii) below. Furthermore, by the previous change of the indices, we can determine the second term in (33). In summary, we conclude the desired formula in (iii).

The following lemma used in the above proof can be obtained from the definitions and elementary calculations, although they are a little complicated.

Lemma 5.5 Let $\mathcal{S}=(\mathcal{C} ; 0)$ be the shadow coloring in Case II as above.

(i) If $p=2$ and $\omega^{q_{1}+q_{2}}=1$, then

$$
\left\langle U_{1}^{q_{1}} U_{2}^{q_{2}+q_{3}} U_{3}^{q_{4}},[\mathcal{S}]\right\rangle=(1+\omega)^{q_{3}+q_{4}} a^{q_{1}+q_{2}+q_{3}+q_{4}} m n / 2 .
$$

(ii) If $\omega^{q_{1}+q_{2}} \neq 1$ and $p=2$ or 3 , then

$$
\left\langle U_{1}^{q_{1}} U_{2}^{q_{2}} U_{3}^{q_{3}+q_{4}}-U_{1}^{q_{1}+q_{2}} U_{2}^{q_{4}} U_{3}^{q_{3}},[\mathcal{S}]\right\rangle=0 .
$$

(iii) If $p=2$ and $\omega^{q_{1}+q_{2}} \neq 1$, then $\left\langle U_{1}^{q_{1}} U_{2}^{q_{2}+q_{3}} U_{3}^{q_{4}},[\mathcal{S}]\right\rangle$ is equal to

$$
\frac{m n}{2} a^{q_{2}+q_{3}}\left(\left(1+\omega^{q_{1}}\right) a^{q_{1}} \delta^{q_{4}}+\left(1+\omega^{q_{4}}\right) a^{q_{4}} \delta^{q_{1}}+\left(1+\frac{\omega^{-q_{1}}+\omega^{-q_{2}}}{1+\omega^{q_{1}+q_{2}}}\right) a^{q_{1}+q_{4}}\right) .
$$

\section{Acknowledgments}

The author thanks Tomotada Ohtsuki and Michihisa Wakui for valuable comments on group cohomologies and 3-manifolds. He is particularly grateful to Yuichi Kabaya for useful discussions and for making several suggestions for improvement. He also expresses his gratitude to the referee for reading this paper carefully. 


\section{Appendix: Proof of Proposition 2.6}

For the proof, we will use the notation $\rho^{k}(g)=g^{k \rho}$ and $\mathcal{K}_{n}$ defined in Section 2.2. Furthermore, for $i \leq n-1$, we set up the following two subsets of the set $\mathcal{K}_{n}$ :

$\mathcal{K}_{n, i}^{+}:=\left\{\left(k_{1}, \ldots, k_{n}\right) \in \mathcal{K}_{n} \mid k_{i}=k_{i+1}+1\right\}, \quad \mathcal{K}_{n, i}^{0}:=\left\{\left(k_{1}, \ldots, k_{n}\right) \in \mathcal{K}_{n} \mid k_{i}=k_{i+1}\right\}$.

Note that $\left|\mathcal{K}_{n, i}^{+}\right|=\left|\mathcal{K}_{n, i}^{0}\right|=t_{X} 2^{n-2}$ and $\mathcal{K}_{n}=\mathcal{K}_{n, i}^{+} \cup \mathcal{K}_{n, i}^{0}$ for any $i \leq n-1$. Hereafter, we denote elements of $\mathcal{K}_{n}$ by $\mathbb{k}_{n}$ for short.

Proof of Proposition 2.6 Our goal is to prove the equality $\partial_{n}^{\mathrm{gr}} \circ \varphi_{n}=\varphi_{n-1} \circ \partial_{n}^{R_{G}}$. For arbitrary $\left(g_{1}, \ldots, g_{n}\right) \in G^{n}$, we start computing the left $\varphi_{n-1} \circ \partial_{n}^{R_{G}}\left(g_{1}, \ldots, g_{n}\right)$ as

$$
\begin{aligned}
\varphi_{n-1} & \left(\sum_{i=1}^{n-1}(-1)^{i}\left(\left(g_{1}, \ldots, g_{i} g_{i+1}, \ldots, g_{n}\right)-\left(g_{1}^{\rho}, \ldots, g_{i-1}^{\rho}, g_{i}^{\rho} g_{i+1}, g_{i+2}, \ldots, g_{n}\right)\right)\right) \\
= & \sum_{i \leq n-1}\left(\sum_{\mathbb{k}_{n} \in \mathcal{K}_{n, i}^{0}}(-1)^{k_{1}-k_{n}+i}\left(g_{1}^{k_{1} \rho}, \ldots, g_{i-1}^{k_{i-1} \rho}, g_{i}^{k_{i+1} \rho} g_{i+1}^{k_{i+1} \rho}, g_{i+2}^{k_{i+2} \rho}, \ldots, g_{n}^{k_{n} \rho}\right)\right. \\
& \left.-\sum_{\mathbb{R}_{n} \in \mathcal{K}_{n, i}^{+}}(-1)^{k_{1}-k_{n}+i+1}\left(g_{1}^{k_{1} \rho}, \ldots, g_{i-1}^{k_{i-1} \rho}, g_{i}^{\left(k_{i+1}+1\right) \rho} g_{i+1}^{k_{i+1} \rho}, g_{i+2}^{k_{i+2} \rho}, \ldots, g_{n}^{k_{n} \rho}\right)\right) \\
= & \sum_{i \leq n-1}\left(\sum_{\mathbb{k}_{n} \in \mathcal{K}_{n}}(-1)^{k_{1}-k_{n}+i}\left(g_{1}^{k_{1} \rho}, \ldots, g_{i-1}^{k_{i-1} \rho}, g_{i}^{k_{i} \rho} g_{i+1}^{k_{i+1} \rho}, g_{i+2}^{k_{i+2} \rho}, \ldots, g_{n}^{k_{n} \rho}\right)\right) .
\end{aligned}
$$

Next we compute the other $\partial_{n}^{\mathrm{gr}} \circ \varphi_{n}\left(g_{1}, \ldots, g_{n}\right)$ as

$$
\begin{aligned}
\partial_{n}^{\mathrm{gr}}( & \left.\sum_{\mathbb{k}_{n} \in \mathcal{K}_{n}}(-1)^{k_{1}-k_{n}}\left(g_{1}^{k_{1} \rho}, \ldots, g_{n}^{k_{n} \rho}\right)\right) \\
= & \sum_{\mathbb{k}_{n} \in \mathcal{K}_{n}}(-1)^{k_{1}-k_{n}}\left(g_{2}^{k_{2} \rho}, \ldots, g_{n}^{k_{n} \rho}\right)+\sum_{\mathbb{k}_{n} \in \mathcal{K}_{n}}(-1)^{k_{1}+n-k_{n}}\left(g_{1}^{k_{1} \rho}, \ldots, g_{n-1}^{k_{n-1} \rho}\right) \\
& +\sum_{i \leq n-1}\left(\sum_{\mathbb{k}_{n} \in \mathcal{K}_{n}}(-1)^{i+k_{1}-k_{n}}\left(g_{1}^{k_{1} \rho}, \ldots, g_{i-1}^{k_{i-1} \rho}, g_{i}^{k_{i} \rho} g_{i+1}^{k_{i+1} \rho}, g_{i+2}^{k_{i+2} \rho}, \ldots, g_{n}^{k_{n} \rho}\right)\right)
\end{aligned}
$$

by definition. Noticing from the previous equation that the third term here is the left $\varphi_{n-1} \circ \partial_{n}^{R_{G}}\left(g_{1}, \ldots, g_{n}\right)$, it suffices to show that the first and second terms vanish. We immediately verify the first vanishing by considering the sum according to $\mathcal{K}_{n}=$ $\mathcal{K}_{n, 1}^{+} \cup \mathcal{K}_{n, 1}^{0}$. Next, for $m \leq t_{X}-1$, we define a subset, $\mathcal{K}_{n, n-1}^{0, m}$, of $\mathcal{K}_{n, n-1}^{0}$ to be 
$\left\{\mathbb{K}_{n} \in \mathcal{K}_{n, n-1}^{0} \mid k_{n}=m\right\}$. Then we can formulate the second term as

$$
\begin{aligned}
\sum_{0 \leq m<t_{X}} & \sum_{\mathbb{k}_{n} \in \mathcal{K}_{n, n-1}^{0, m}}(-1)^{k_{1}-k_{n}+n}\left(\left(g_{1}^{k_{1} \rho}, \ldots, g_{n-1}^{k_{n-1} \rho}\right)-\left(g_{1}^{\left(k_{1}+1\right) \rho}, \ldots, g_{n-1}^{\left(k_{n-1}+1\right) \rho}\right)\right) \\
= & \sum_{0 \leq m<t_{X}} \sum_{\mathbb{k}_{n} \in \mathcal{K}_{n, n-1}^{0,0}}(-1)^{k_{1}-k_{n}+n}\left(\left(g_{1}^{\left(k_{1}+m\right) \rho}, \ldots, g_{n-1}^{\left(k_{n-1}+m\right) \rho}\right)\right. \\
= & \sum_{\mathbb{k}_{n} \in \mathcal{K}_{n, n-1}^{0,0}}(-1)^{k_{1}-k_{n}+n}\left(\left(g_{1}^{k_{1} \rho}, \ldots, g_{n-1}^{k_{n-1} \rho}\right)-\left(g_{1}^{\left(k_{1}+t_{X}\right) \rho}, \ldots, g_{n-1}^{\left(k_{n-1}+t_{X}\right) \rho}\right)\right)
\end{aligned}
$$

Since $\rho^{t_{X}}(g)=g$ for any $g \in G$, this term is zero as desired.

\section{References}

[1] S Asami, K Kuga, Colorings of torus knots and their twist-spuns by Alexander quandles over finite fields, J. Knot Theory Ramifications 18 (2009) 1259-1270 MR2569560

[2] KS Brown, Cohomology of groups, corrected 2nd edition, Graduate Texts in Mathematics 87, Springer, New York (1982) MR1324339

[3] J S Carter, D Jelsovsky, S Kamada, L Langford, M Saito, Quandle cohomology and state-sum invariants of knotted curves and surfaces, Trans. Amer. Math. Soc. 355 (2003) 3947-3989 MR1990571

[4] J S Carter, S Kamada, M Saito, Geometric interpretations of quandle homology, J. Knot Theory Ramifications 10 (2001) 345-386 MR1825963

[5] F J-B J Clauwens, The adjoint group of an Alexander quandle arXiv:1011.1587

[6] R Dijkgraaf, E Witten, Topological gauge theories and group cohomology, Comm. Math. Phys. 129 (1990) 393-429 MR1048699

[7] M Eisermann, Quandle coverings and their Galois correspondence, to appear in Fundamenta Mathematicae

[8] R Fenn, C Rourke, B Sanderson, Trunks and classifying spaces, Appl. Categ. Structures 3 (1995) 321-356 MR1364012

[9] R Fenn, C Rourke, B Sanderson, The rack space, Trans. Amer. Math. Soc. 359 (2007) 701-740 MR2255194

[10] A Inoue, Quandle homomorphisms of knot quandles to Alexander quandles, J. Knot Theory Ramifications 10 (2001) 813-821 MR1840269

[11] A Inoue, Y Kabaya, Quandle homology and complex volume (2013) arXiv: 1012. 2923v3 
[12] D Joyce, A classifying invariant of knots, the knot quandle, J. Pure Appl. Algebra 23 (1982) 37-65 MR638121

[13] Y Kabaya, Cyclic branched coverings of knots and quandle homology, Pacific J. Math. 259 (2012) 315-347 MR2988494

[14] A Kawauchi, A survey of knot theory, Birkhäuser, Basel (1996) MR1417494

[15] D Kraines, Massey higher products, Trans. Amer. Math. Soc. 124 (1966) 431-449 MR0202136

[16] I J Leary, The mod-p cohomology rings of some p-groups, Math. Proc. Cambridge Philos. Soc. 112 (1992) 63-75 MR1162933

[17] J Mandemaker, Various topics in rack and quandle homology, Master thesis, Radboud University (2009)

[18] J Milnor, On the 3-dimensional Brieskorn manifolds $M(p, q, r)$, from: "Knots, groups, and 3-manifolds (Papers dedicated to the memory of R H Fox)", (L P Neuwirth, editor), Ann. of Math. Studies 84, Princeton Univ. Press (1975) 175-225 MR0418127

[19] T Mochizuki, The 3-cocycles of the Alexander quandles $\mathbb{F}_{q}[T] /(T-\omega)$, Algebr. Geom. Topol. 5 (2005) 183-205 MR2135551

[20] T Nosaka, On quandle homology groups of Alexander quandles of prime order, Trans. Amer. Math. Soc. 365 (2013) 3413-3436 MR3042590

[21] T Nosaka, Quandle cocycles from invariant theory, Adv. Math. 245 (2013) 423-438 MR3084434

[22] T Nosaka, Homotopical interpretation of link invariants from finite quandles (2014) arXiv: $1210.6528 \mathrm{v} 5$

[23] M Wakui, On Dijkgraaf-Witten invariant for 3-manifolds, Osaka J. Math. 29 (1992) 675-696 MR1192735

Faculty of Mathematics, Kyushu University

744, Motooka, Nishi-ku, Fukuoka 819-0395, Japan

nosaka@math.kyushu-u.ac.jp

Received: 9 February $2013 \quad$ Revised: 8 October 2013 
